\title{
An equivalent calculation method for 3D contact area between two rough surfaces based on 2D profiles
}

Jianping Jing ( $\sim$ jianpj@sjtu.edu.cn )

Shanghai Jiao Tong University

\section{Wen Meng}

Shanghai Jiao Tong University

\section{Dai Zezeng}

Shanghai Jiao Tong University

\section{Mei Qing}

Hunan Aviation Powerplant Research Institute, Aero Engine Corporation of China

\section{Yuan Wei}

Hunan Aviation Powerplant Research Institute, Aero Engine Corporation of China

\section{Research Article}

Keywords: Two rough surfaces, Oblique and peak-peak contact, Real contact area and angle, Numerical simulation

Posted Date: February 23rd, 2022

DOI: https://doi.org/10.21203/rs.3.rs-1338190/v1

License: (9) (1) This work is licensed under a Creative Commons Attribution 4.0 International License. Read Full License 


\title{
An equivalent calculation method for 3D contact area between two rough surfaces based on 2D profiles
}

\author{
Wen Meng $^{1}$, Dai Zezeng ${ }^{1}$, Jing Jianping ${ }^{1 *}$, Mei Qing $^{2}$ and Yuan Wei ${ }^{2}$ \\ ${ }^{1}$ The State Key Laboratory of Mechanical System and Vibration, Shanghai Jiao Tong University, \\ Shanghai, 200240, China. \\ ${ }^{2}$ Hunan Aviation Powerplant Research Institute, Aero Engine Corporation of China, Zhuzhou, \\ 412002, China.
}

*Corresponding author(s). E-mail(s): jianpj@sjtu.edu.cn; Contributing authors: manyouzhe@sjtu.edu.cn; daizezeng@sjtu.edu.cn; 372575800@qq.com; gucao_2000@163.com;

\begin{abstract}
The calculation of the real contact area between rough surfaces is an important prerequisite for studying the contact characteristics of mechanical joints. However, it is challenging to accurately calculate the real contact area between rough surfaces by existing theoretical and experimental methods. Therefore, an efficient and practical equivalent calculation method for the real contact area between three-dimensional(3D) rough surfaces based on two-dimensional(2D) profiles is presented in this paper. The main advantage of the presented method as compared with those available in the literature is that the real contact area can be predicted only by the section of the $3 \mathrm{D}$ rough surfaces in contact, which greatly reduces the computational cost. The sampling strategy of sections is studied to improve the accuracy of the proposed method. The accuracy of the proposed equivalent calculation method is verified by numerical simulation. The results show that the proposed equivalent calculation method can efficiently predict the real contact area between rough surfaces within the scope of engineering application.
\end{abstract}

Keywords: Two rough surfaces, Oblique and peak-peak contact, Real contact area and angle, Numerical simulation

\section{Graphical Abstract}

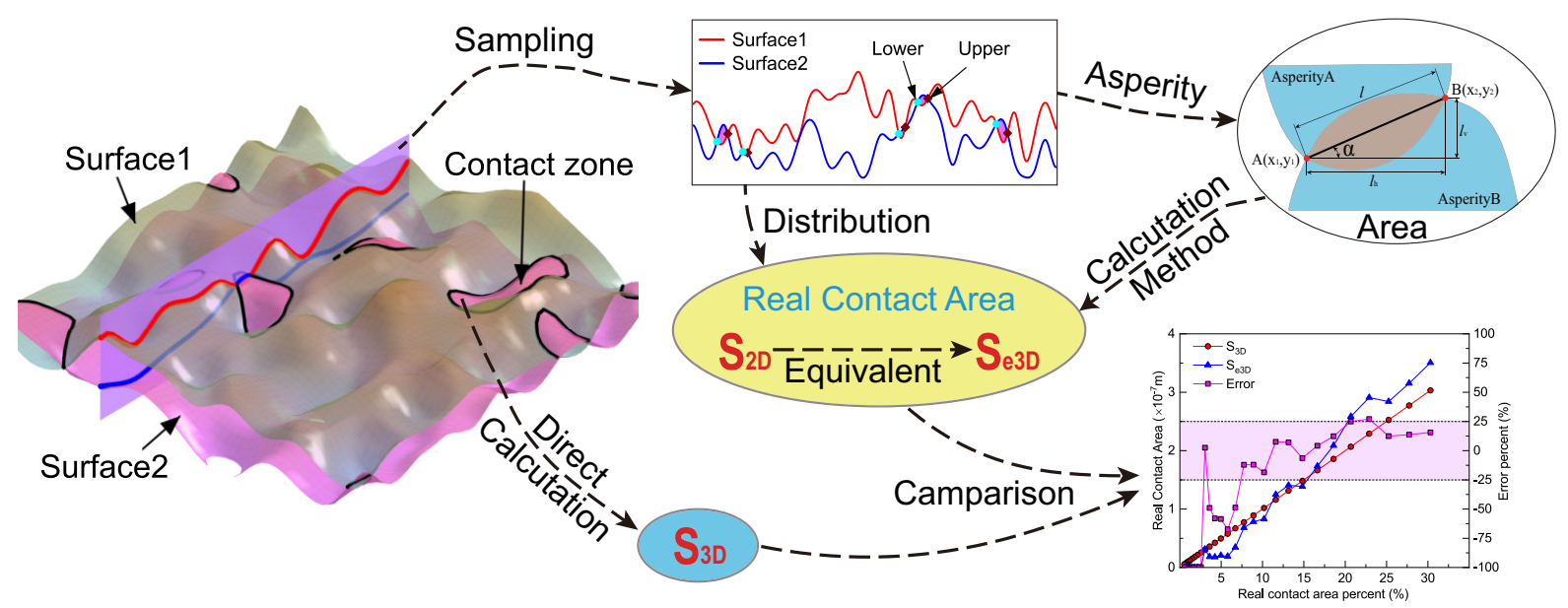




\section{Introduction}

The surface of all objects in the physical world is made up of rough surfaces covered with numerous asperities of different sizes, which has been proven by many experiments [1-3]. The contact surface is formed by squeezing two rough surfaces and is widely present on all mechanical interfaces, plays an important role in contact characteristics on the mechanical interfaces (e.g. stiffness [4], damping [5], slip [6, 7], wear [8, 9], stickiness [10], lubrication [11]) and the overall characteristics of mechanical systems (e.g. inherent frequencies [12], modal vibrations [13]). In recent years, many scholars have studied the contact characteristics between rough surfaces, including the characterization of rough surface micromorphology [14, 15], contact mechanics model [16], the characteristics of contact stiffness and damping $[17,18]$.

The true or real contact area between two rough surfaces, which is much smaller than the nominal contact area, is the basis for studying the contact mechanics of the rough surface and affects the dynamic properties of the contact surface [19]. Therefore, the study of the calculation method for real contact area has aroused the wide interest of the scientific community [20-25]. The theoretical calculation methods of the real contact area mainly include the analytical methods and the numerical methods.

\section{Analytical methods}

The analytical methods usually hold that the real contact area can be obtained by the contact area integration of asperities. The multiscale model based on asperities is a typical analytical method, originally proposed by Greenwood [26, 27], which usually assumes that parameters such as the height of asperities on rough surfaces are subject to statistical distributions (e.g. Gaussian). Combined with Hertz's theory, the relationship between the normal deformation and the contact area between asperities can be calculated, and then the characteristics between rough surfaces are calculated by the integration of asperities. The GW model assumes that asperities on rough surfaces can be reduced to hemispheres with the same radius, which is significantly different from the experimental observations in recent years [28]. Therefore, many scholars have developed modified models based on GW models, such as CEB model [29], Brake model [30], WA model [31]. These models have corrected the distribution of height and deformation stage of asperities respectively, which are more in line with the micromorphological characteristics between the real rough surfaces, and improve the accuracy of the prediction for the real contact area between rough surfaces, but the gap with the actual situation is significant due to the existence of many assumptions. In addition, the parameters used in multiscale methods can be influenced by factors such as the resolution of the measuring instrument and the length of sampling. Therefore, there methods have limitations [32].

The fractal method is another alternative analytical method, which assumes that rough surfaces can be superimposed by numerous sine curves, and then assumes that the contact area with each other's asperities is subject to the island area distribution function [33-35]. Subsequently, the two-variable W-M function is proposed by Yan $[36,37]$, which can better describe the microscopic appearance of the real 3D physical surface. Because of the dimensional independence of the fractal method, it can better characterize the microscopic appearance of the real rough surface, has been used by many scholars to characterize the rough surface [38, 39] and mechanics modeling $[5,16,40-42]$. However, the maximum contact area between asperities is calculated iteratively for the fractal method according to the pressure load of the rough surface and the total contact load, and then the true contact area between the rough surfaces is obtained according to the integration of the island area distribution function [40]. Therefore, the real contact area is obtained by the contact load test, there is no corresponding theoretical derivation. In addition, the island area distribution function is based on the distribution of the earth's island, and there is little public information to prove whether the contact area between asperities is subject to the island area distribution, which makes the real contact area calculated based on fractal theory uncertain.

Many scholars have revised the island area distribution function based on the M-B model. Komvopoulos [20] solves the problem of non-zero domain intervals in traditional M-B models by the domain extension factors. Based on the true geometry of the sliding rail roller, $\mathrm{Li}$ [41] obtains the contact area distribution function between asperities with geometric properties. Chen $[43,44]$ considers the geometry of the contact between the two spheres and corrects the area distribution function of asperities in which the two surfaces come into contact by a correction factor. Because the deformation state of asperities in traditional MB models is changing from plasticity to elasticity, which is in contrast to classical mechanics laws, Morag [45] proposed a multiscale fractal contact mechanics model, which divides asperities into different scales according to the contact area, and then assuming that the contact area between asperities on different scales is in line with the island area distribution. Finally, all scales are summed up to obtain the total contact load and the contact area between rough surfaces [46-48]. This solves the problem of deformation state transformation of asperities in fractal theory, but still faces the accuracy problem of the contact area distribution hypothesis. The Persson model is another analytical model, which holds that the contact pressure of rough surfaces obeys a specific probability distribution, which has been studied by many scholars [49-51].

Numerical methods 
Many scholars have proposed rough texture generation methods. Yan[36] proposed a method for generating the isotropic and anisotropic rough surfaces based on the power spectrum method. Uchidate[52] presents a noncausal 2D autoregressive model for generating 3D random topography datasets with periodic boundaries. Yang[53] proposed a numerical method with controllable autocorrelation length for generating different Gaussian isotropic surfaces. Yoshida[54] studied the influence of tip radius on measurement results in contact roughness. Qu[55] proposed a facile method for simulating rough membrane surface morphology. Pawlus[14] summarized random rough surface generation methods and compared the characteristics of various methods. The method of rough surface generation based on fractal theory is widely used because it can easily generate the microscopic morphology similar to the actual engineering surface.

Thanks to the rapid development of computer technology, various numerical simulation methods have emerged, such as the Finite Element Model (FEM) [56-58], Boundary Element Method (BEM) [59, 60], the Green's Function Molecular Dynamics (GFMD) [22, 61], the Boundary Element Method based on Rapid Fourier Transformation (FFT-BEM) [62]. Numerical methods usually discrete rough surfaces into a matrix of nodes arranged according to certain rules, and then determine whether the discrete nodes or elements are in contact according to the distance, stress, and other parameters, and then calculate the real contact area according to the number of discrete nodes or elements in contact with each other. The computational accuracy of numerical simulation methods depends heavily on the number of discrete elements. Typically, the smaller sample steps or the larger number of elements, the more accurate the true contact area is. However, a common problem for the traditional numerical simulation method of $3 \mathrm{D}$ rough surfaces contact is the exponential growth of computational requirements. In other words, the amount of computation increases exponentially with the number of elements, which greatly increases the computational cost of numerical simulation. The literature has been found to show that the largest number of units is [60,63], the author used the mesh with $4096 \times 4096$ elements for the Green function molecular dynamics. While this improves the computational accuracy of the real contact area, it makes the computational cost tens of millions of elements, which in many cases is almost unacceptable.

Therefore, some methods for accurately calculating the real contact area by relatively sparse elements are proposed $[19,64]$. Based on the distribution law of discrete mesh calculation error, Yastrebov [65, 66] proposes a correction formula for calculating the real contact area. This method can converge to a certain curve with high precision by relatively few meshes and reduces the computational cost of the real contact area by numerical simulation method. However, the method still has an exponential growth relationship between the number of sampling points and the cost of calculation. Especially when the real contact area is small, the calculation error of a single element will increase dramatically, at which point increasing the number of sampling points becomes the only option. With limited computing power, the results of the calculation of the real contact area may be unreliable.

In general. There are too many assumptions in the analytical method, so the calculated real contact area may be far from the actual situation. The accurate calculation of the real contact area in numerical methods is costly and unacceptable in many cases. In addition, whether the analytical or numerical simulation, it is generally assumed that the contact between rough surfaces can be reduced to an equivalent rough surface and a rigid smooth plane (Equivalent-Plane contact), which ignores the oblique contact between rough surfaces, but few studies have verified the rationality of this hypothesis.

\section{Objectives and outline}

In this paper, an equivalent 3D contact area calculation method based on 2D profiles is proposed, which can be used for numerical methods to calculate the real contact area between two 3D rough surfaces. The advantage of the presented method is that the calculated cost is linear with the number of sampling elements (the calculation cost is $n$ ), which greatly reduces the computational requirements compared to the traditional 3D numerical method (the computational cost is $n^{2}$ ). Thus, it has the potential to extend the real contact area between rough surfaces to nano or even smaller scales.

The structure of the rest of this article. In section 2, the fractal method is used to generate the contact model between 2D and 3D rough surfaces. In section 3, the method of calculating the contact area and angle between $2 \mathrm{D}$ rough surfaces by the differential method is proposed, and then the area and angle distribution characteristics of 2D Equivalent-Plane contact and 2D oblique contact are compared. In section 4, The calculation methods of the contact area and angle between the 3D equivalent rough surface and the plane and the ones between two 3D rough surfaces are proposed, and the accuracy of the calculation methods is verified. In section 5 , the relationship between sampling steps and the calculation error of real contact area is studied in 2D and 3D simulation. In section 6 , the real contact area calculation method for the equivalent 3D rough surface based on 2D profiles is derived, and the sampling strategy of $2 \mathrm{D}$ profiles is improved. Then, the accuracy of the equivalent $3 \mathrm{D}$ contact area calculation method is studied. In section 7 , the main conclusions of this paper are summarized. 


\section{Rough surface generation}

\section{$2.13 \mathrm{D}$ rough surface}

For the 3D rough surface, Yan and Komvopoulos [36] propose a two-variable function Eq.(1), which can quickly generate the isotropic and anisotropic 3D rough surface. In this paper, the function is adopted to generate the 3D contact between two rough surfaces, which is shown in Fig.1. It can be seen from Fig.1(b), which is the partially enlarged view of Fig.1(a), that the oblique contact is common between two rough surfaces.

$$
\begin{aligned}
z(x, y)= & L\left(\frac{G}{L}\right)^{(D-2)}\left(\frac{\ln \gamma}{M}\right)^{1 / 2} \sum_{m=1}^{M} \sum_{n=n_{\min }}^{n_{\max }} \gamma^{(D-3) n} \times\left\{\cos \phi_{m, n}\right. \\
& \left.-\cos \left[\frac{2 \pi \gamma^{n}\left(x^{2}+y^{2}\right)^{1 / 2}}{L} \times \cos \left(\tan ^{-1}\left(\frac{y}{x}\right)-\frac{\pi m}{M}\right)+\phi_{m, n}\right]\right\}
\end{aligned}
$$

where $z(x, y)$ and $L$ is the height of $\operatorname{asperity}(\mathrm{m})$ and sampling length $(\mathrm{m})$, respectively. $D$ is the fractal dimension $(2<$ $D<3), G$ is the characteristic scale factor(m), $\phi$ is a random phase $(0<\phi<2 \pi), \gamma$ is a parameter that determines the frequency density of the profile(chosen to be 1.5 in this paper), $n_{\min }$ and $n_{\max }$ is the min and max sampling frequency, respectively. $n_{\min }=\log (L) / \log (\gamma), n_{\max }=\log (L s) / \log (\gamma) \cdot M$ denotes the number of superposed ridges on surface.

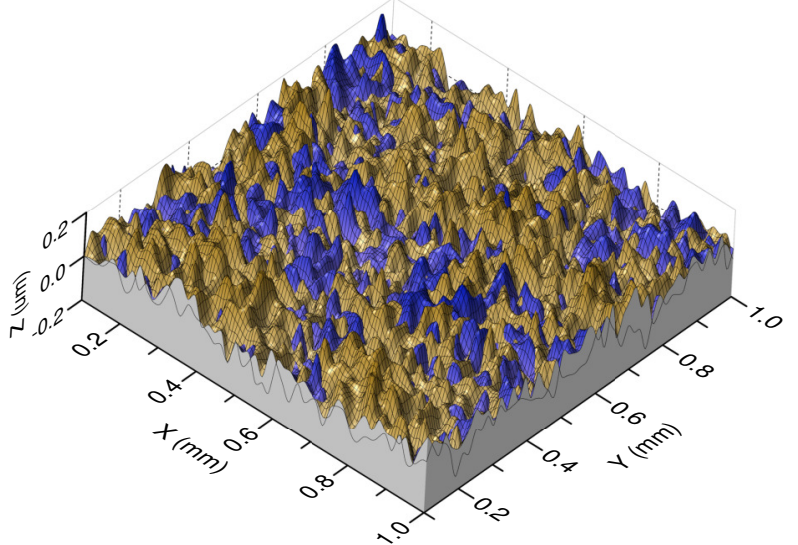

(a)

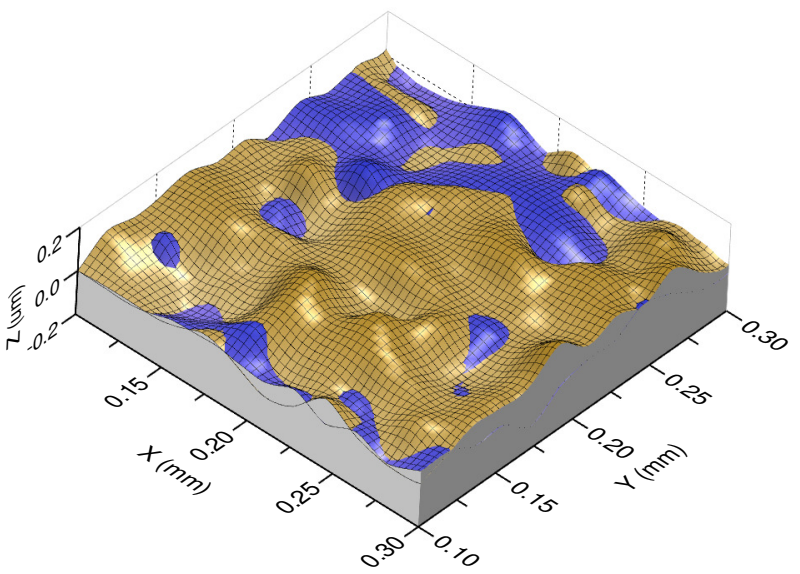

(b)

Fig. $13 \mathrm{D}$ contact between two rough surfaces, (b) is the partial enlarged view of (a). $\left(D_{1}=D_{2}=2.3, G_{1}=G_{2}=1.2 \times 10^{-10} \mathrm{~m}\right)$

\section{$2.22 \mathrm{D}$ rough surface}

Numerous studies assume that the 2D cross-sectional profile of an isotropic rough surface has similar morphological characteristics to its 3D rough surface[40]. For the 2D rough surface, Yan and Komvopoulos [36] derived a singlevariable model based on the modified $\mathrm{W}$-M function, which was given in Eq.(2). In this paper, the 2D contact between two rough surfaces is shown in Fig.2, which shows most contact points exist on the shoulders of asperities while rare one contacts each other exactly from peak to peak.

$$
z(x)=L\left(\frac{G}{L}\right)^{D-1}(\ln \gamma)^{1 / 2} \sum_{n=n_{\min }}^{n_{\max }} \gamma^{(D-2) n}\left[\cos \phi_{1, n}-\cos \left(\frac{2 \pi \gamma^{n} x}{L}-\phi_{1, n}\right)\right] .
$$

where $z(x)$ is the height of asperity $(\mathrm{m}), D$ is the fractal dimension $(1<D<2)$, the meaning of other parameters is similar to the $3 \mathrm{D}$ function Eq.(1). 


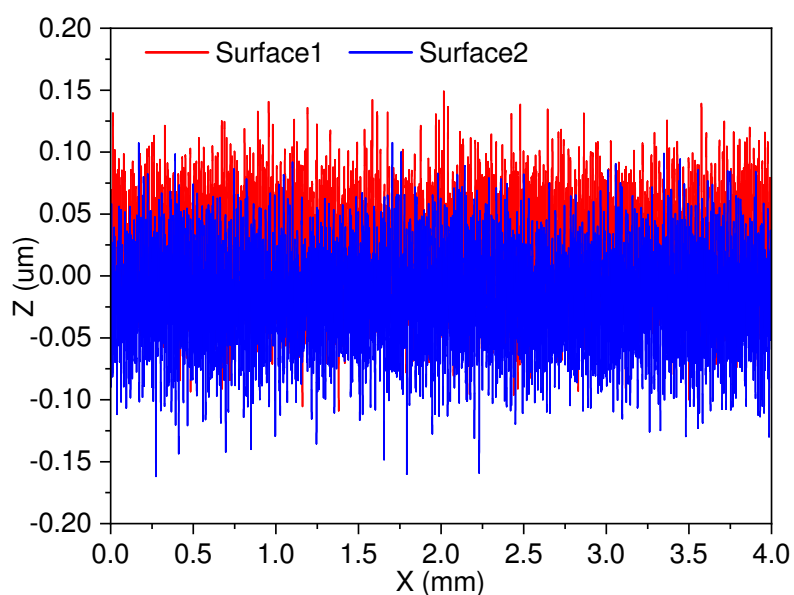

(a)

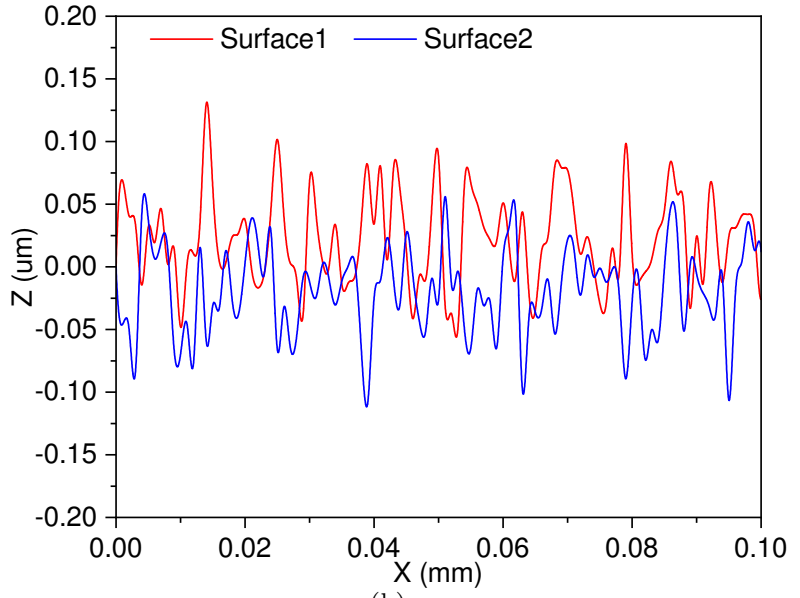

(b)

Fig. 2 2D contact between two rough surfaces, (b) is the partial enlarged view of (a). $\left(D_{1}=D_{2}=1.3, G_{1}=G_{2}=1.2 \times 10^{-10} \mathrm{~m}\right)$

\section{Comparison of 2D peak and oblique contact}

\subsection{The contact area calculation method between $2 \mathrm{D}$ rough surfaces}

The contact between two rough surfaces can be modeled as an equivalent rough surface in contact with a rigid smooth surface [16, 40], which is widely used in traditional methods is shown in Fig.3(a). The fundamental principle for contact area calculation in traditional methods is as follows. First, the horizontal crossing points between the traditional equivalent rough surface and the rigid smooth surface are calculated by numerical simulation method. Secondly, the transversal length $l_{h}$ between two rough surfaces is obtained by calculating the distance between two ends. Finally, the sectional area of contact zone is $S_{h}=\pi\left(l_{h} / 2\right)^{2}$. The contact zone is simplified as a horizontal circular zone in traditional methods, which can be observed from Fig.3(a). However, the contact zone between two rough surfaces is oblique in the real world, shown as Fig.3(b). The proposed method can obtain the oblique contact area, which is more consistent with the actual contact condition between two rough surfaces. The fundamental principle for contact area calculation in the proposed method is as follows. First, the oblique crossing points are obtained by numerical simulation. After then, the oblique transversal length $l$ is obtained by calculating the distance between two oblique crossing points. Finally, the oblique intersection area is $S_{2 D}=\pi(l / 2)^{2}$.
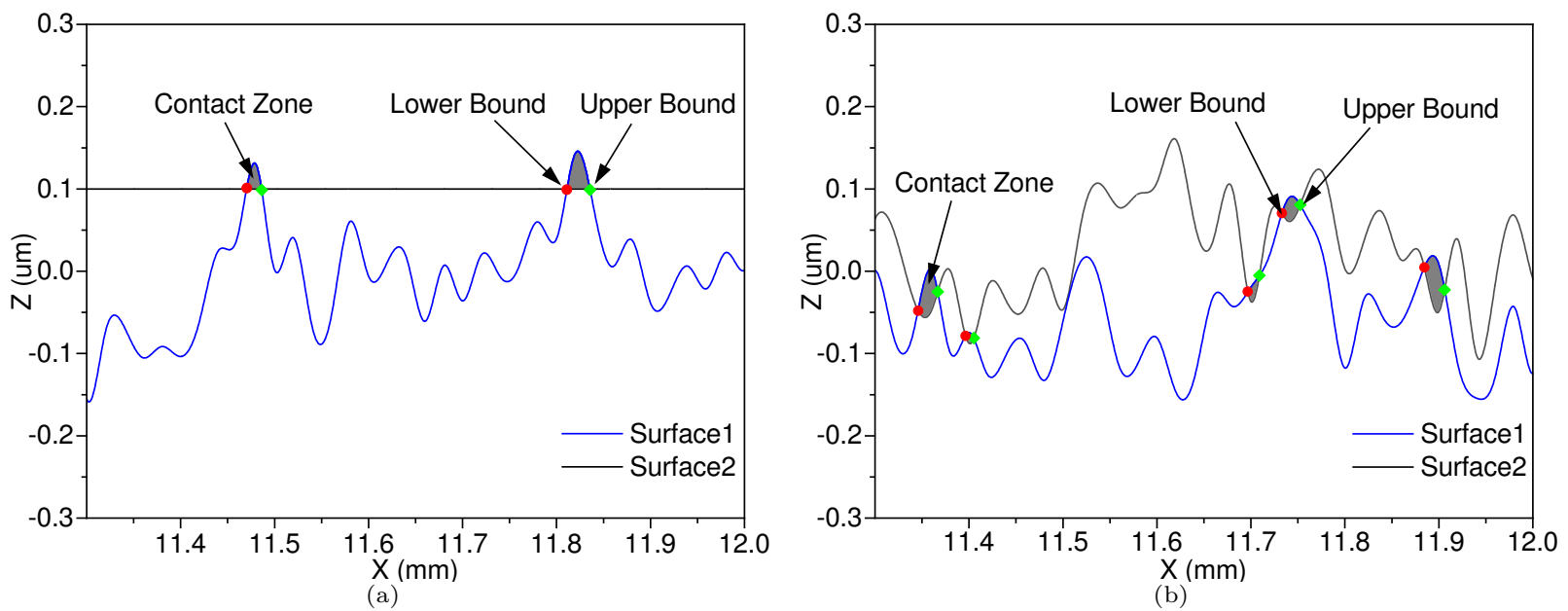

Fig. 3 The principle of $2 \mathrm{D}$ contact zone area calculation, (a) The traditional equivalent rough surface contact, (b) The real rough surface contact.

The contact area, which is obtained by the traditional method, is the horizontal projection of the real contact area, such as Fig.4. However, the contact area $S_{2 D}$ calculated by the proposed method, which is the vector sum of horizontal contact area $S_{h}$ and vertical contact area $S_{v}$, is the true reflection of what is in the real world. Due to 


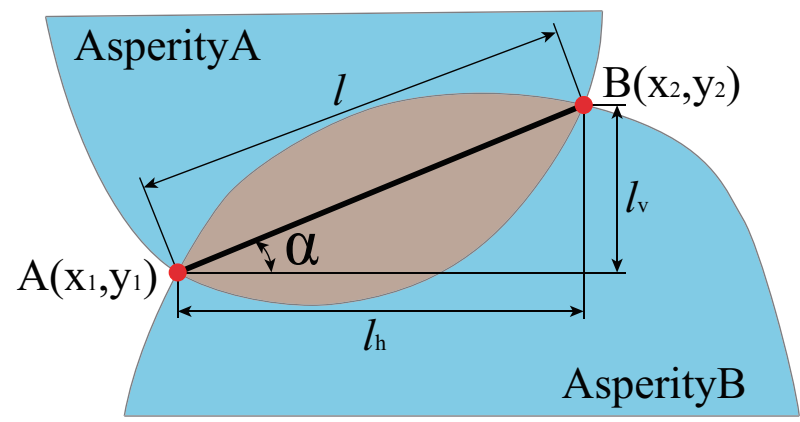

Fig. 4 The oblique contact area calculation method. $l$ is the oblique length of the two end points of the contact area between the asperities, $l_{h}$ and $l_{v}$ are the lengths of the horizontal and vertical projections of $l$, respectively. $\alpha$ is the inclined contact angle between asperities.

the horizontal and vertical contact mechanical property can be reflected simultaneously by the contact area $S_{2 D}$, the proposed method has an advantage over the traditional method in $2 \mathrm{D}$ contact between two rough surfaces. The relationship between the traditional method and the proposed method in this paper is:

$$
\begin{aligned}
S_{2 D}= & \sqrt{S_{h}^{2}+S_{v}^{2}} \\
= & \sqrt{\pi\left(l_{h} / 2\right)^{2}+\pi\left(l_{v} / 2\right)^{2}} \\
= & \frac{\sqrt{\pi}}{2} \sqrt{\left(x_{2}-x_{1}\right)^{2}+\left(y_{2}-y_{1}\right)^{2}} \\
& \alpha_{2 D}=\arctan \left(\frac{y_{2}-y_{1}}{x_{2}-x_{1}}\right)
\end{aligned}
$$

where $S_{2 D}$ and $\alpha_{2 D}$ are the oblique contact area and angle of the $2 \mathrm{D}$ rough profiles, respectively. $S_{h}$ is the horizontal contact area, $S_{v}$ is the vertical contact area.

\subsection{Verification of contact area calculation method}

The 2D contact area calculation methods can be classified as the equivalent single rough surface contact and the two rough surfaces contact. The calculation result of the former is shown in Fig.5(a), and one of the latter is shown in Fig.5(b). The results demonstrate that the crossing points between two $2 \mathrm{D}$ rough surfaces can be accurately identified by the proposed methods in the paper, which ensure that the contact area is accurately calculated.

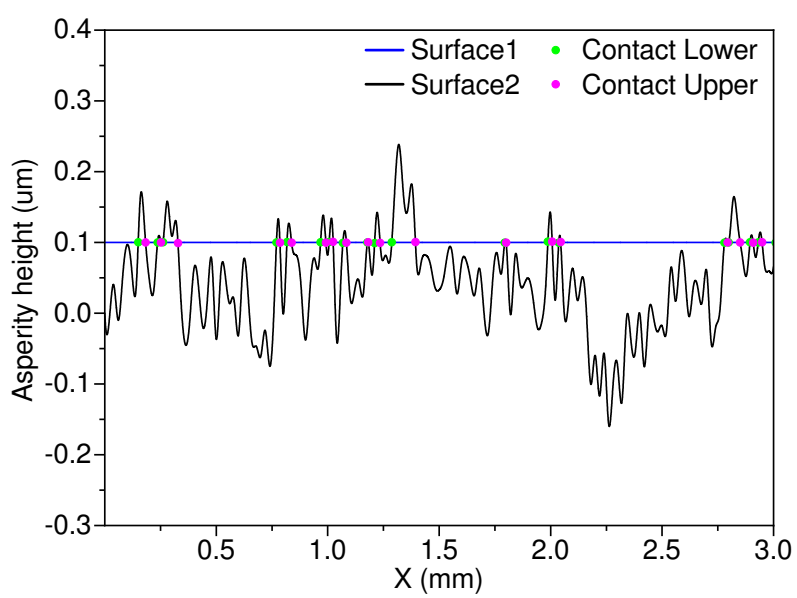

(a)

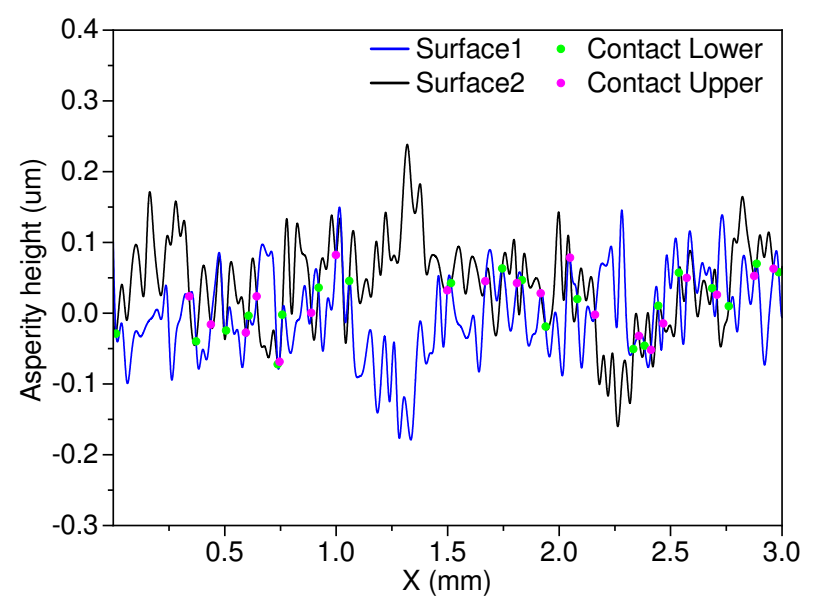

(b)

Fig. 5 The verification of $2 \mathrm{D}$ contact area calculation method, (a) Equivalent-Plane contact, (b) Contact between two rough surfaces. 


\subsection{Comparison of $2 \mathrm{D}$ contact area and angle distribution}

The contact area and angle of 2D peak and oblique contact are calculated based on the methods described above in this section, which are shown in Fig.6. The fractal dimension $D$ and characteristic scale factor $G$ are 1.3 and $1.2 \times 10^{-10} \mathrm{~m}$, respectively. The sampling length $L=1 \mathrm{~mm}$. The resolution $L s$ of Fig.6 (a)-(c), (d)-(f) and (g)-(i) are $1 \times 10^{-5} \mathrm{~m}, 1 \times 10^{-6} \mathrm{~m}$ and $1 \times 10^{-7} \mathrm{~m}$, respectively.

Fig.6(a), (d) and (g) are the frequency distribution statistics of the contact area for peak and oblique contact. It can be seen from Fig.6(a) (d) and (g) that the contact area distribution of the peak and oblique contact is basically the same when resolution $L s=1 \times 10^{-5} \mathrm{~m}$. However, the difference in contact area distribution of the peak and oblique contact gradually increases when the resolution $L s$ is $1 \times 10^{-6} \mathrm{~m}$ and $1 \times 10^{-7} \mathrm{~m}$. This may be due to the increase in resolution that makes the larger asperities subdivide into smaller-sized asperities, which increases the proportion of the oblique contact asperities, thereby increasing the difference of the peak and oblique contact in area distribution.

Fig.6(b), (e) and (h) are the lollipop graph of contact area in the peak and oblique contact. The blue point represents the real contact area of the asperity in oblique contact, and the pink point represents the contact area of the asperity in contact with the corresponding peak. The smaller the distance between these two points, the smaller the area difference between the oblique contact and the peak contact. It can be seen that when the resolution $L s$ increases, the number of the asperities and the proportion of smaller-sized asperities at the same time. In addition, when the resolution increases, the difference in the contact area between oblique and peak contact also gradually increases, which proves the correctness of the aforementioned inference.
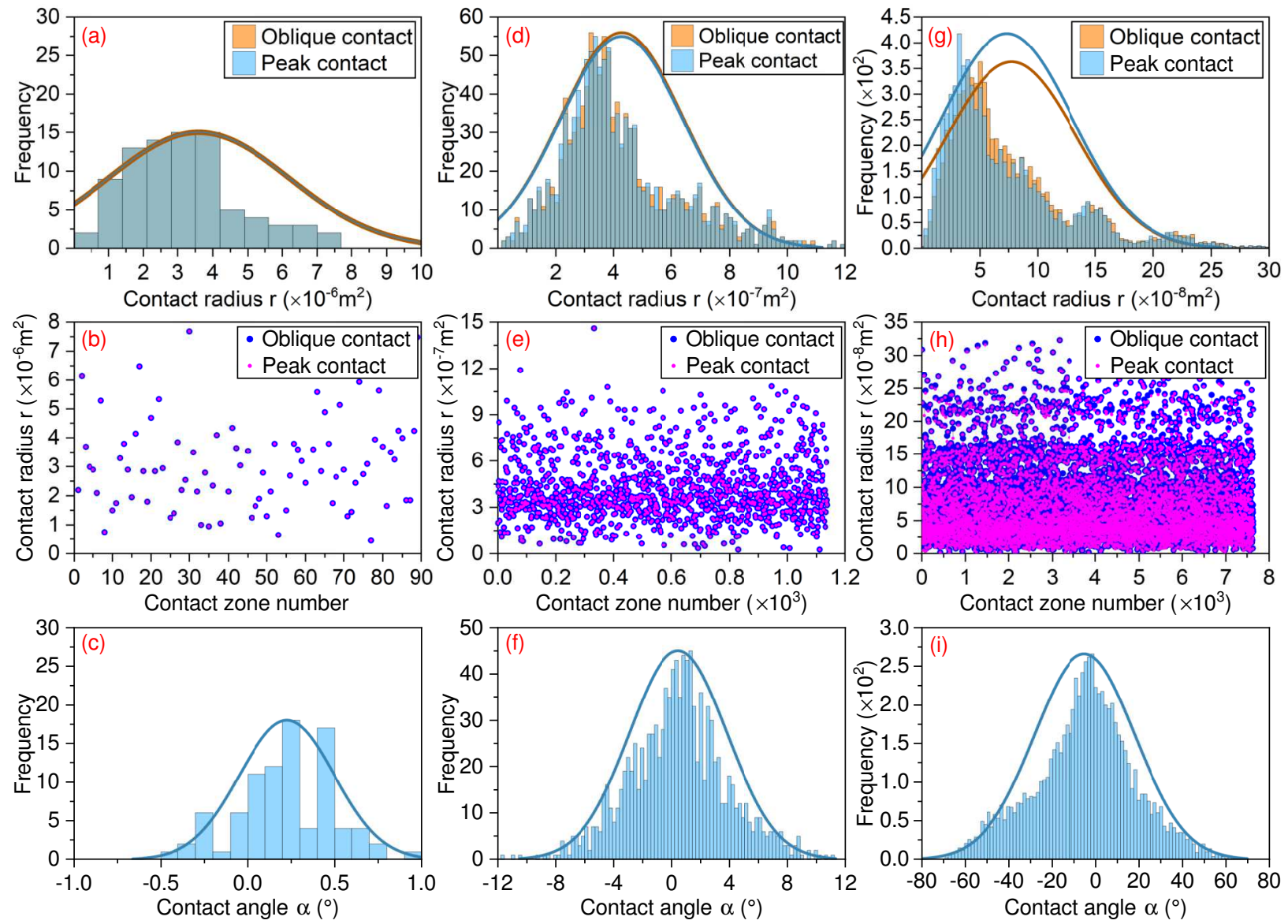

Fig. 6 Comparison of the $2 \mathrm{D}$ peak and oblique contact area

Fig.6(c), (f) and (i) are the frequency distribution of the contact angle $\alpha$ for the oblique contact. It can be seen from Fig.6(c) that the maximum contact angle $\alpha_{\max }$ is 1 degree when the resolution is $1 \times 10^{-5} \mathrm{~m}$. However, when the resolution are $1 \times 10^{-6} \mathrm{~m}$ and $1 \times 10^{-7} \mathrm{~m}$, the maximum contact angle $\alpha_{\max }$ are 12 and 80 degrees respectively. Combined with the distribution of the contact area between asperities, it further proves the aforementioned inference that the increase in resolution increases the number of smaller asperities, thereby increasing the value of the 
maximum contact angle $\alpha_{\max }$ and the proportion of asperities with larger contact angles. In addition, it can be seen that as the resolution increases, the symmetry of contact angle between two isotropic rough surfaces concerning 0 degrees gradually increases.

\subsection{Analysis of 2D contact force}

Fig.6(c, f, and i) demonstrate that the symmetry of the 2D contact angle gradually increases when the sampling resolution of the isotropic rough surface increases. According to the symmetry of contact angle on the 2D rough surface, a reasonable assumption can be made: For any existing contact angle $\alpha_{i}$, there is a corresponding contact angle $\beta_{i}$ of the same size and opposite direction, which is shown as Fig.7. So, the relationship between the contact angle of the asperities is:

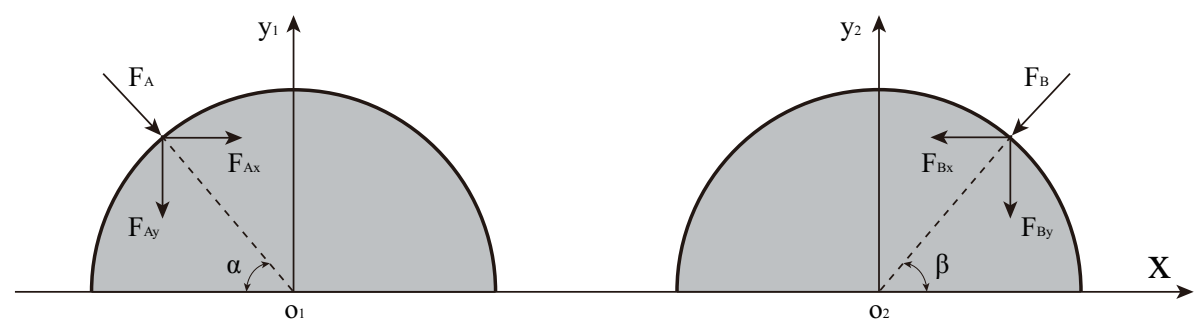

Fig. 7 The force diagram of asperity

$$
\begin{aligned}
& F_{A i}+F_{B i}=\left[\begin{array}{ll}
F_{A x i}+F_{B x i} & F_{A x i}+F_{B x i}
\end{array}\right] \\
&= {\left[\begin{array}{ll}
0 & F_{A i} \sin \alpha_{i}+F_{B i} \sin \beta_{i}
\end{array}\right] } \\
& F_{n}=\sum_{i=1}^{m}\left(F_{A i} \sin \alpha_{i}+F_{B i} \sin \beta_{i}\right)
\end{aligned}
$$

where $F_{n}$ is the normal contact load on the surface, $\mathrm{m}$ is the number of asperity on the sample surface, $F_{A i}$ and $F_{B i}$ is the force of asperity whose sum of contact angles $\alpha_{i}$ and $\beta_{i}$ is 0 . Due to $F_{A x i}+F_{B x i}=0$, the horizontal components of the load on the $2 \mathrm{D}$ rough surface profile can cancel each other out. The normal components of the load on all asperities are superimposed on each other, which is equal to the normal load on the rough surface.

Therefore, it can be concluded that the oblique contact between two 2D rough surfaces can be simplified to peak-peak contact when the tangential load is not considered.

\section{The calculation method of oblique contact area between two 3D rough surfaces}

\subsection{The contact area calculation method between two $3 \mathrm{D}$ rough surfaces}

Similar to the contact between two $2 \mathrm{D}$ rough surfaces, the equivalent rough surface assumption is used widely in traditional 3D rough surface contact, which is shown in Fig.8(a). This is similar to the MB model, the corresponding area $S_{x o y}$ in traditional methods is the horizontal projection of actual contact area $S_{3 D}$. However, the true contact situation is shown in Fig.8(b), whose intersecting curve is space closed curves. Therefore, a 3D oblique contact area calculation method is proposed in this paper, which can catch the space characteristics of contact area between two 3D rough surfaces, as shown in Fig.9. Assuming that the asperities on two rough surfaces in contact with each other can be simplified as shown in Fig.9(a), and their contact area can be projected on three coordinate planes, which is shown in Fig.9(b).

Due to the effective calculation of the corresponding area for 3D space surface being relatively complicated and little practical physical meaning, the projection method is used to calculate the projection area $S_{x o y}, S_{y o z}$ and $S_{x o z}$ of the space asperity contact area $S_{3 D}$ on three Cartesian coordinate planes, respectively. So the contact area between two $3 \mathrm{D}$ asperities $S_{3 D}$ is:

$$
S_{3 D}=\sqrt{\left(S_{x o y}\right)^{2}+\left(S_{x o z}\right)^{2}+\left(S_{y o z}\right)^{2}}
$$




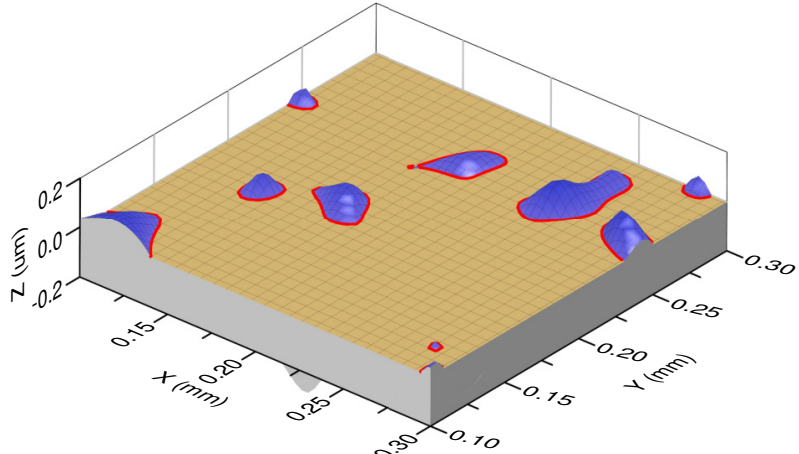

(a)

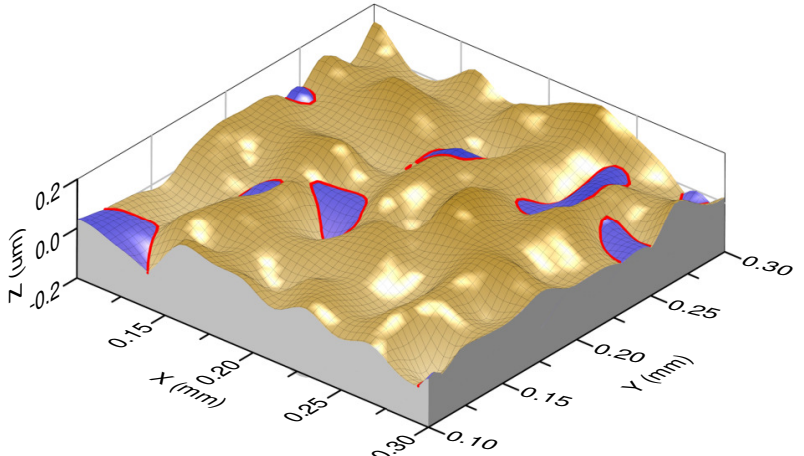

(b)

Fig. 8 Comparison of 3D equivalent contact and two rough surfaces contact, (a) Example of contact between equivalent 3D rough surface and rigid smooth surface, (b) Example of contact between two 3D rough surfaces.

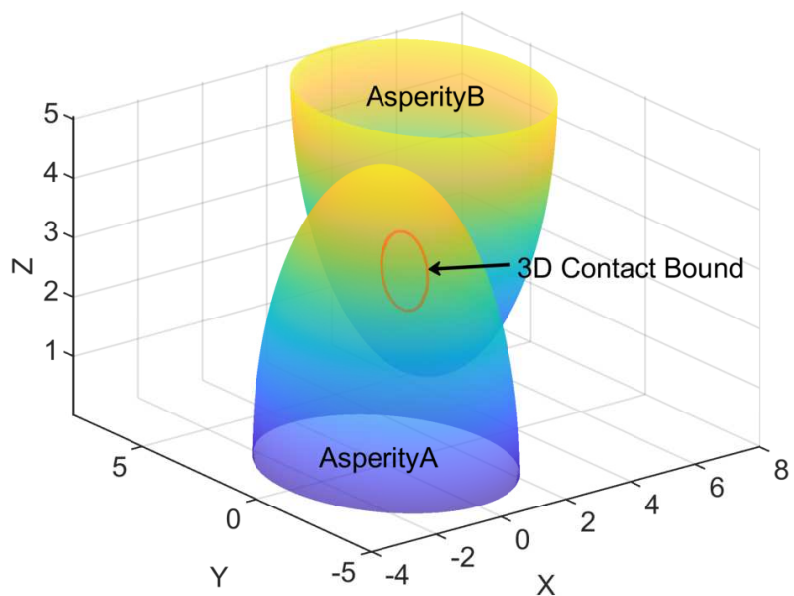

(a)

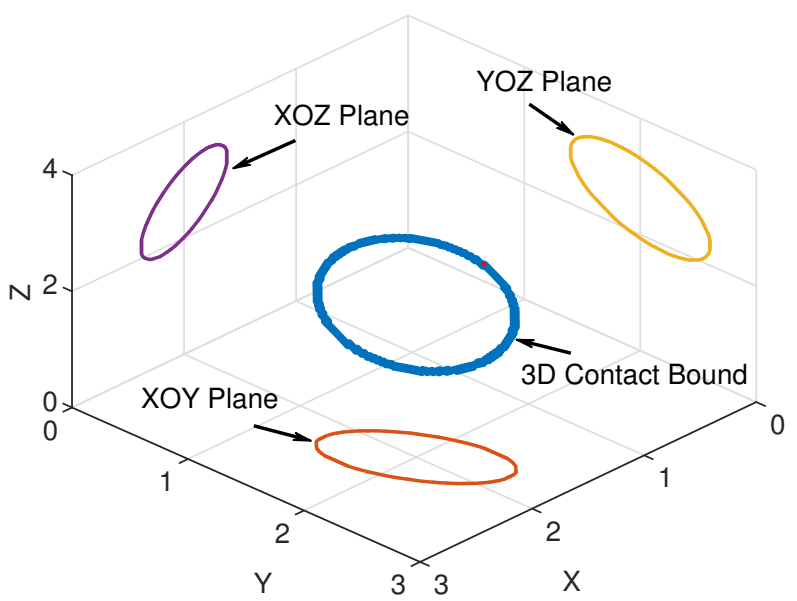

(b)

Fig. 9 The principle of three-dimension contact zone identification, (a) Contact example of corresponding asperities on two rough surfaces, (b) Example of projecting oblique contact zone on three Cartesian coordinate planes.

Then the angle vector $\alpha_{i}$ between the oblique contact section of the $i$-th asperity and the three Cartesian coordinate planes can be calculated:

$$
\alpha_{i}=\left[\begin{array}{lll}
\alpha_{x o y i} & \alpha_{y o z i} & \alpha_{x o z i}
\end{array}\right]=\left[\arccos \left(\frac{S_{x o y}}{S_{3 D}}\right) \arccos \left(\frac{S_{y o z}}{S_{3 D}}\right) \arccos \left(\frac{S_{x o z}}{S_{3 D}}\right)\right]
$$

where $\alpha_{x o y i}, \alpha_{y o z i}$ and $\alpha_{x o z i}$ are the angles between the $i$-th asperity and the three coordinate planes respectively.

Many studies have shown that the $2 \mathrm{D}$ section profile of any isotropic 3D rough surface has the same physical properties as the original 3D rough surface. According to subsection 3.4, the contact angle between the 2D sections for two isotropic 3D rough surfaces is symmetrical with respect to 0 degrees. That is to say, the $2 \mathrm{D}$ oblique contact can be simplified as an equivalent profile contacting a rigid plane when only the normal load is considered. Therefore, it can be inferred that the contact between two isotropic 3D rough surfaces can be simplified to the contact between an equivalent 3D rough surface and a rigid smooth surface when only the normal load is considered. In other words, the normal contact characteristics of two rough surfaces can be effectively expressed by the contact between an equivalent rough surface and a smooth rigid plane. Since only considers the normal contact characteristics in this article, it only needs to calculate the projected area $S_{x o y}$ of the oblique contact area $S_{3 D}$ on the XOY coordinate plane.

An image processing method is adopted to calculate the contact area between two 3D rough surfaces. Firstly, the commercial software MATLAB is used to generate two 3D rough surfaces based on the fractal theory Eq.1. Secondly, the contact model between two rough surfaces, whose top view such as Fig.10(a) is obtained for image processing, is established based on the distance between the mean surface of two rough surfaces. Thirdly, the number of the pixels $N_{c}$ in the contact zone is calculated by the color difference between two rough surfaces, which 
is shown in Fig.10(b). Finally, the 3D real contact area $S_{\text {xoy }}$ can be expressed as:

$$
S_{\text {xoy }}=\frac{N_{c}}{N_{\text {total }}} L_{a} L_{b}=\frac{N_{c}}{n_{a} n_{b}} L_{a} L_{b}
$$

where $n_{a}$ and $n_{b}$ are the pixels number in the length and width directions of the sample image, respectively. $N_{\text {total }}$ is the total number of pixels in the sample image. $L_{a}$ and $L_{b}$ are the actual physical length and width of the sample, respectively.

Fig.10(a) shows that the two 3D rough surfaces should be identified, and the blue zone is higher than the yellow zone, so the intersecting curve between them is the intersecting section boundary curve. Fig.10(b) is the identification result, and it demonstrates that the proposed method is effective for boundary curve identification of contact zone between two $3 \mathrm{D}$ rough surfaces.

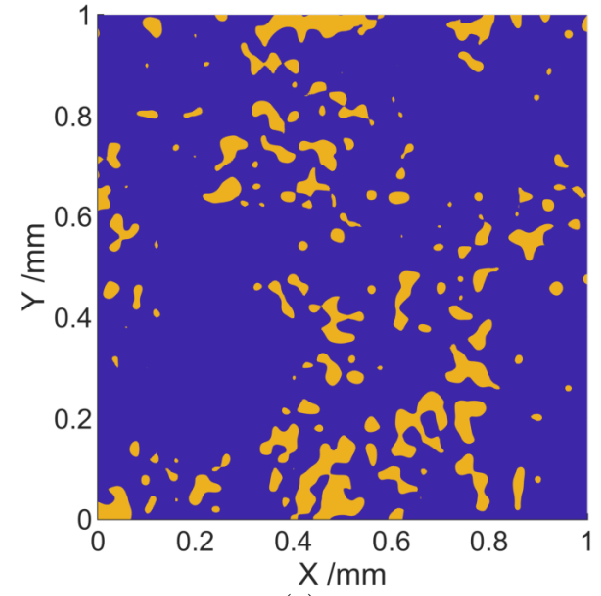

(a)

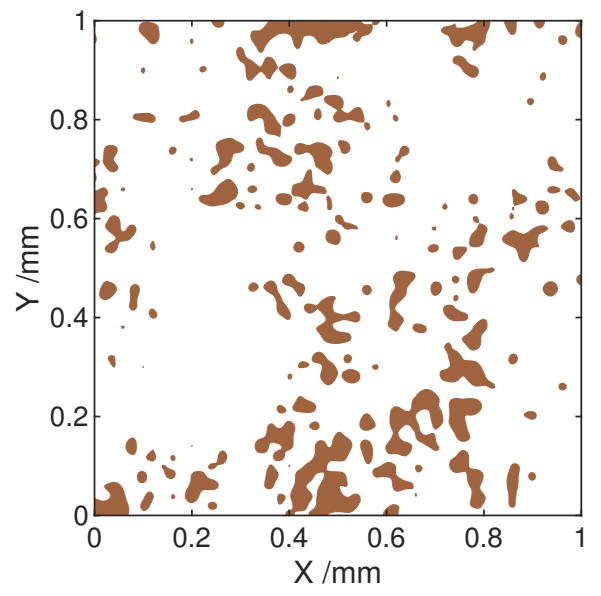

(b)

Fig. 10 The principle for obtaining the contact area of two 3D rough surfaces, (a) The XOY projection of two rough surfaces contact zone, (b) The calculation result of the contact area in the XOY projection.

\subsection{Verification of $3 \mathrm{D}$ contact area calculation method}

To verify the correctness of the 3D contact area calculation method proposed in Section 4.1, which is used to identify the boundary of the contact area in this section, and then the recognition result is superimposed with the original image, which is shown in Fig.11(b). It can be seen from the figure that the contact area calculation method between two 3D rough surfaces proposed in this paper can effectively identify the boundary of the contact area and ensure the calculation accuracy. This verifies the accuracy of the 3D contact area calculation method proposed in the text from the side perspective.

\section{The relationship between sampling step and calculation accuracy}

According to the fractal theory, the mechanical interface can be subdivided to the atomic scale, and the roughness of which is observed from different scales is significantly different, so the contact area between rough surfaces calculated under different observation scales is also different. Studies have shown that the smaller the observation scale, the smaller the real contact area between rough surfaces. However, there are limits to the computing power of computer simulation systems in real life, so it is impossible to increase the resolution indefinitely. To ensure the accuracy of the calculation while reducing the requirements for the computing power of the computer system as much as possible, the relationship between the sampling step and the calculation error under different scales will be studied in this section.

\section{$5.12 \mathrm{D}$ numerical simulation}

The top view of the intersection between two $2 \mathrm{D}$ curves can be viewed as segments of different lengths on a straight line, which are the length of the intersection zone. Therefore, the numerical simulation of the contact between 2D curves can be simplified as the intersection of the line and the circle as shown in Fig.12. 


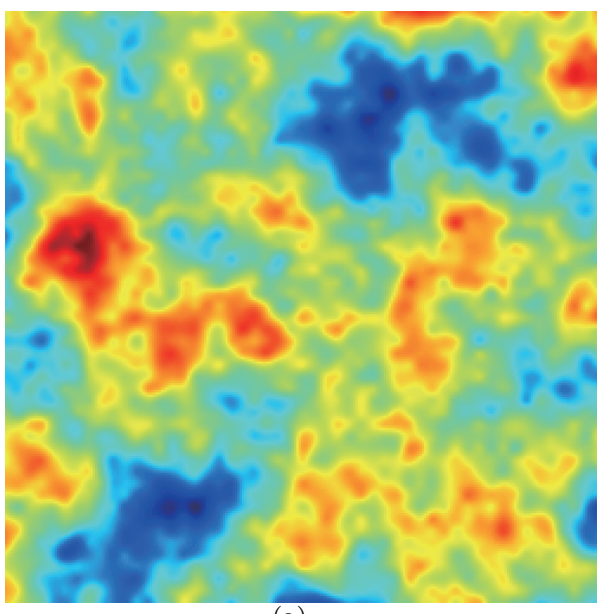

(a)

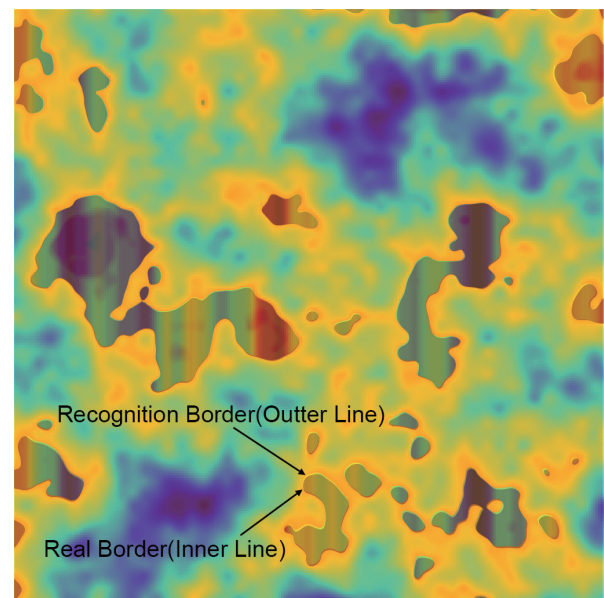

(b)

Fig. 11 The verification of three-dimension contact zone calculation method, (a) Equivalent rough surface for identification, (b) Recognition results of boundary for the contact area between two rough surfaces.
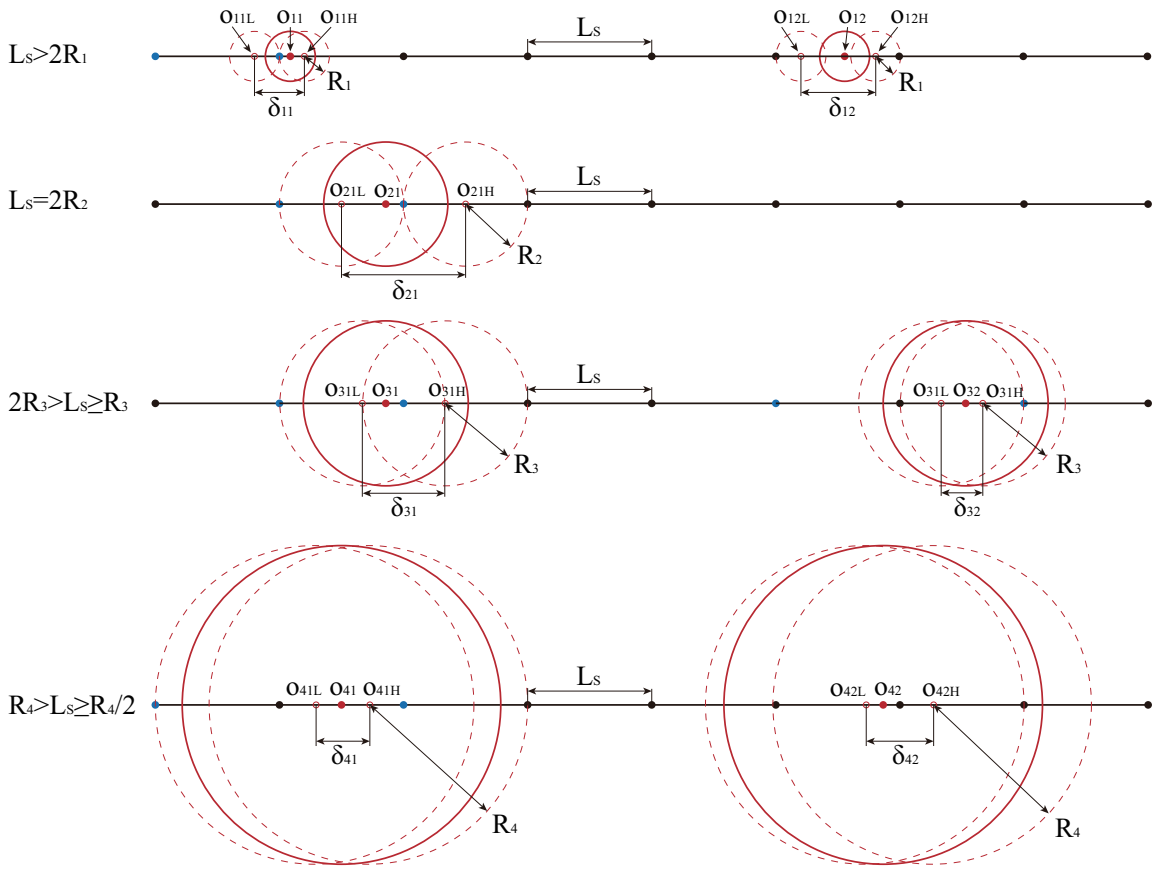

Fig. 12 Schematic diagram of the contact area between asperities obtained in 2D simulation.

The surface of objects in the real world contains asperities of different scales, while the sampling length $L_{s}$ in numerical simulation is often uniform or has a minimum limit. Assuming that the minimum value of the sampling step is $L_{s}$, there must be asperities with diameters $2 R$ greater than, equal to, and smaller than the sampling length $L_{s}$ in the numerical simulation. These situations will be discussed separately in the following.

- $L_{s}>2 R$

Suppose the radius of asperities, whose diameter is less than $L_{s}$, is $R_{1}$. At this point, the asperities in the numerical simulation are detected with probability $P_{1}$ :

$$
P_{1}=\frac{\delta_{11}}{L_{s}}=\frac{2 R_{1}}{L_{s}}
$$


Therefore, the mathematical expectation of the contact area between asperities obtained by the $2 \mathrm{D}$ numerical simulation can be expressed as $E\left(a_{1}\right)$ :

$$
E\left(a_{1}\right)=P_{1} \times \pi\left(\frac{L_{s}}{2}\right)^{2}=\pi \frac{R_{1} L_{s}}{2}
$$

Therefore, the calculation error $E_{r}\left(a_{1}\right)$ of contact area between asperities in 2D numerical simulation and its ratio $E_{p}\left(a_{1}\right)$ are:

$$
\begin{gathered}
E_{r}\left(a_{1}\right)=E\left(a_{1}\right)-\pi R_{1}^{2}=\pi\left(\frac{R_{1} L_{s}}{2}-R_{1}^{2}\right) \\
E_{p}\left(a_{1}\right)=\frac{E_{r}\left(a_{1}\right)}{\pi R_{1}^{2}}=\frac{L_{s}}{2 R_{1}}-1
\end{gathered}
$$

Because $L_{s}$ is greater than $2 R_{1}, E_{r}\left(a_{1}\right)>0, E_{p}\left(a_{1}\right) \in(0, \infty)$.

- $L_{s}=2 R$

Suppose the radius of asperities, whose diameter is equal to $L_{s}$, is $R_{2}$. The probability that the asperities being detected is $P_{2}=1$. Therefore, the calculation error of contact area between asperities in 2D numerical simulation is $E_{r}\left(a_{2}\right)$ :

$$
E_{r}\left(a_{2}\right)=E\left(a_{2}\right)-\pi R_{2}^{2}=\pi\left[\left(L_{s} / 2\right)^{2}-R_{2}^{2}\right]=0
$$

Therefore, the expected error percentage $E_{p}(a 2)$ of the contact area between asperities calculated by $2 \mathrm{D}$ simulation is 0 .

- $2 R>L_{s}>R$

In this case, the internal contact area between asperities may contain one or two discrete points. The mathematical probabilities are $P_{31}$ and $P_{32}$ respectively ( $R_{3}$ is the contact area between asperities):

$$
\begin{gathered}
P_{31}=\frac{2\left(L_{s}-R_{3}\right)}{L_{s}} \\
P_{32}=1-P_{31}=\frac{\left(2 R_{3}-L_{s}\right)}{L_{s}}
\end{gathered}
$$

The mathematical expectation $E\left(a_{3}\right)$ of the contact area between asperities is:

$$
\begin{aligned}
E\left(a_{3}\right) & =P_{31} \times \pi\left(\frac{2 L_{s}}{2}\right)^{2}+P_{32} \times \pi\left(\frac{3 L_{s}}{2}\right)^{2} \\
& =\frac{\pi L_{s}}{4}\left(10 R_{3}-L_{s}\right)
\end{aligned}
$$

The calculation error $E_{r}\left(a_{3}\right)$ of contact area between asperities in 2D numerical simulation is:

$$
E_{r}\left(a_{3}\right)=E\left(a_{3}\right)-\pi R_{3}^{2}=\pi\left(\frac{10 R_{3} L_{s}}{4}-\frac{L_{s}^{2}}{4}-R_{3}^{2}\right)
$$

The expected error percentage $E_{p}(a 3)$ of the contact area between asperities calculated by $2 \mathrm{D}$ simulation is:

$$
E_{p}\left(a_{3}\right)=\frac{E_{r}\left(a_{3}\right)}{\pi R_{3}^{2}}=\frac{10 L_{s}}{4 R_{3}}-\left(\frac{L_{s}}{2 R_{3}}\right)^{2}-1
$$

According to the relationship between $L_{s}$ and $R_{3}$, it can be obtained that $E_{r}\left(a_{3}\right)>0, E_{p}\left(a_{3}\right) \in(1.25,3)$.

Based on the above derivation process, the expected error value $E_{r}\left(a_{i}\right)$ and its percentage $E_{p}\left(a_{i}\right)$ of the contact area between the larger-sized asperities obtained by simulation can be calculated. It can be seen from the above derivation formula that the mathematical expectation $E_{r}\left(a_{i}\right)$ of the contact area between the asperities calculated by the $2 \mathrm{D}$ numerical simulation is larger than the actual value except when the diameter $2 R$ of the asperities is 
exactly equal to the integer multiple of the sampling step $L_{s}$. When the diameter $2 R$ of the asperities is smaller than the sampling step $L_{s}$, the expected error percentage $E_{p}\left(a_{i}\right)$ of the calculated contact area between asperities may be close to 0 , but the upper limit of the error percentage is infinite. This is exactly in line with experience that fewer asperities may go undetected, which results in an infinity error percentage. That is to say, it is uncontrollable. On the other hand, it is assumed that the diameter of the asperities is larger than the sampling step. If the asperity diameter is an integer multiple of the sampling step, the expected error percentage of the simulated contact area is zero. Otherwise, the expected error percentage is a small positive interval. This shows that when the diameter of the asperities is larger than the sampling step, the calculation error of the contact area between the asperities obtained by the $2 \mathrm{D}$ simulation can be kept within a certain range. That is to say, it is controllable. In fact, the expected error percentage gradually decreases as the diameter of the asperities increases. Therefore, if it is necessary to accurately measure the contact area between asperities with small diameters, reducing the sampling interval may be the most direct and effective way.

\subsection{D numerical simulation}

The top view of the contact zone between two 3D rough surfaces can be viewed as a $2 \mathrm{D}$ plane containing multiple closed contact zones, which is shown in Fig.13. Similar to 2D numerical simulation, many 3D numerical simulation methods sample a plurality of criss-cross 2D sampling point arrays to jointly judge the contact zones between asperities. The blue points in Fig.13 are the boundary points of the contact zones identified by the 3D numerical simulation method, and then the contact area of the corresponding blue zones in the figure can be obtained.

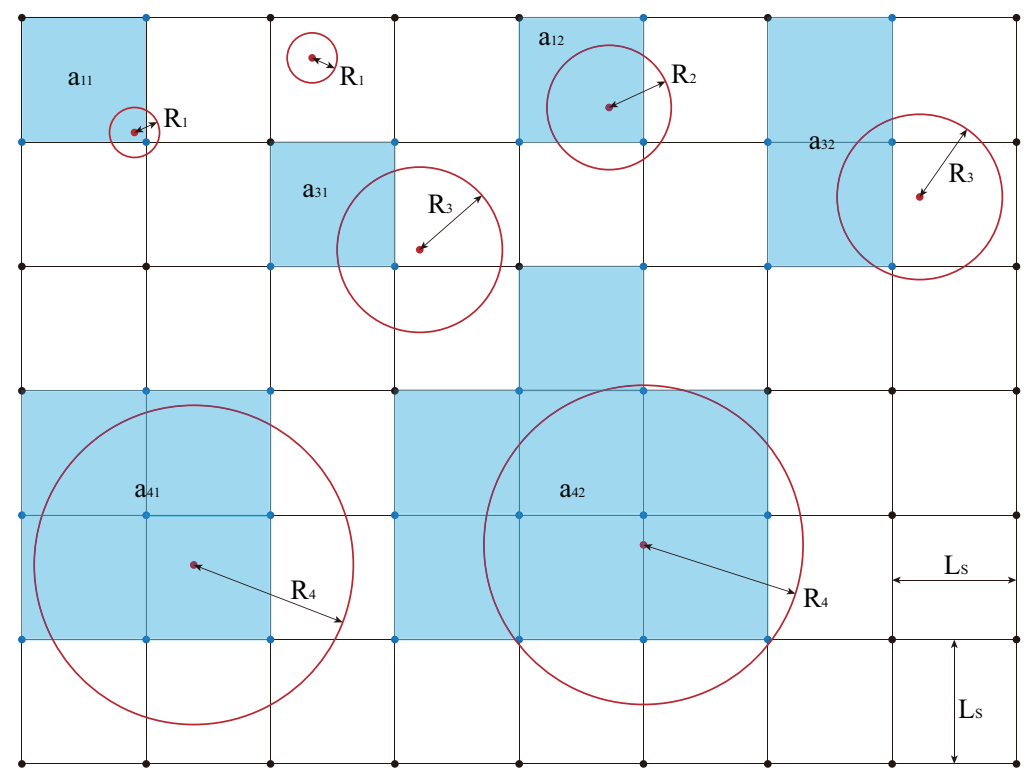

Fig. 13 Schematic diagram of the contact area between asperities obtained in 3D simulation.

As can be seen from Fig.13, if the diameter $2 R$ of asperities is smaller than the sampling step $L_{s}$, the asperities may also fail to be detected, and the calculated value of the contact area between the detected asperities is also greater than the real value, which is the same as the rule in $2 \mathrm{D}$ simulation. The inference method in section 5.1 can also be used to deduce the influence rule of the relative size of sampling step on the calculation accuracy in 3D numerical simulation. However, the analysis of the error rule in 3D numerical simulation is much more complicated than that in 2D simulation, so this paper will not carry out the reasoning in detail. Similar conclusions to $2 \mathrm{D}$ numerical simulations can be obtained only by qualitative analysis. That is to say, when the diameter $2 R$ of the contact area between the asperities is less than $1.128 L_{s}$ (Calculated by the mesh area being equal to the contact area between asperities), the error percentage of the numerical simulation increases sharply, which may be uncontrollable. When the diameter of the contact area between the asperities is greater than $1.128 L_{s}$, the error percentage of the numerical simulation gradually decreases, which is controllable.

Therefore, there must be a certain minimum sampling resolution $L_{s}$ for any given scale of rough surface contact, which makes the calculation error of the real contact area between asperities greater than or equal to this scale within a controllable range. However, the calculation error of the contact area between asperities smaller than this 
scale will increase exponentially. It can be concluded that the calculation error of the actual contact area between asperities tends to infinity when the contact area between asperities tends to be infinitely small from the resolution of the measuring instrument or the step length of numerical simulation sampling. That is to say, when the actual contact area between rough surfaces is small enough, the contact area calculated by simulation method may be unreliable. Only the contact area between asperities whose contact radius is larger than the sampling resolution can be calculated with a certain degree of credibility.

\section{The equivalent calculation method of contact area between two 3D surfaces based on $2 \mathrm{D}$ profiles}

In this section, a method for calculating the contact area between 3D rough surfaces based on 2D profiles is proposed. It can equivalently calculate the real contact area and angle between two 3D rough surfaces by the profiles of the $2 \mathrm{D}$ cross-section of the $3 \mathrm{D}$ rough surface.

\subsection{The equivalent calculation principle for 3D contact area}

For a sufficiently large isotropic rectangular rough surface sample, whose length and width are $L_{a}$ and $L_{b}$ respectively, assume that its arbitrary 2D cross-section profile $l$ should have the same physical properties as the 3D rough surface sample, which is shown in Fig.14. If the number and length of asperities passed by the section line are $N$ and $S_{i}$ respectively, and the equivalent contact area $S_{e 3 D}$ between the rectangular isotropic rough surface sample can be expressed as:

$$
\begin{aligned}
S_{e 3 D} & =L_{a} \frac{N}{L_{a}} \times L_{b} \frac{N}{L_{a}} \times \frac{\sum_{i=1}^{N} S_{i}}{N} \\
& =\frac{L_{b} N}{L_{a}} \times \sum_{i=1}^{N} S_{i}
\end{aligned}
$$

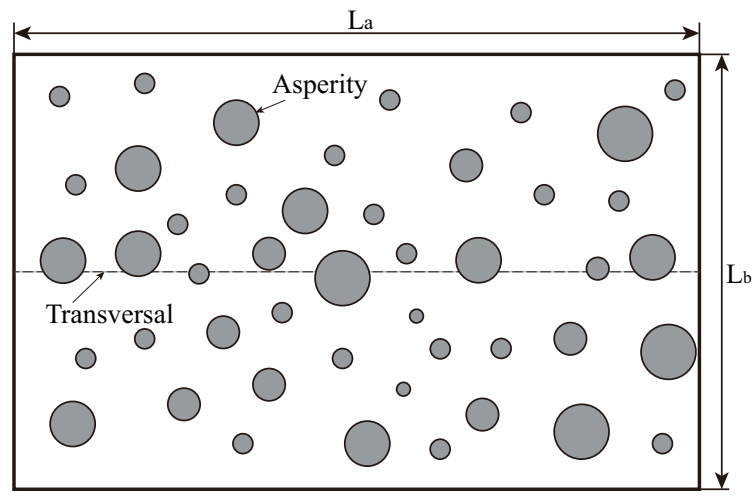

Fig. 14 Schematic diagram of rough surface profile cross-section sampling

Since the calculation cost of the contact between two 3D rough surfaces increases greatly with the increase of the scale, the sampling strategy of the $2 \mathrm{D}$ rough profile corresponding to the $3 \mathrm{D}$ rough surface has been further improved, which is shown in Fig.15.

The basic principle is to sample multiple 2D profiles at equal intervals in the orthogonal direction, and then calculate the average value of the number and area of their contact points, and finally obtain the equivalent 3D real contact area between two rough surfaces. This improvement measure not only increases the effective sampling length, but also obtains the contact characteristics between the entire 3D rough surfaces more comprehensively. If the number and length of asperities passed by the section line are $N$ and $S_{i}$ respectively, and it can be considered that the number $N_{u}$ and area $S_{u}$ of asperities per unit length for isotropic rough surface are:

$$
\begin{gathered}
N_{u}=\frac{\sum_{j=1}^{n} N_{j}}{n L_{a}} \\
S_{u}=\left(\sum_{j=1}^{n} \sum_{i=1}^{m_{j}} \pi\left(l_{i j} / 2\right)^{2}\right) \times \frac{1}{\sum_{j=1}^{n} N_{j}}
\end{gathered}
$$



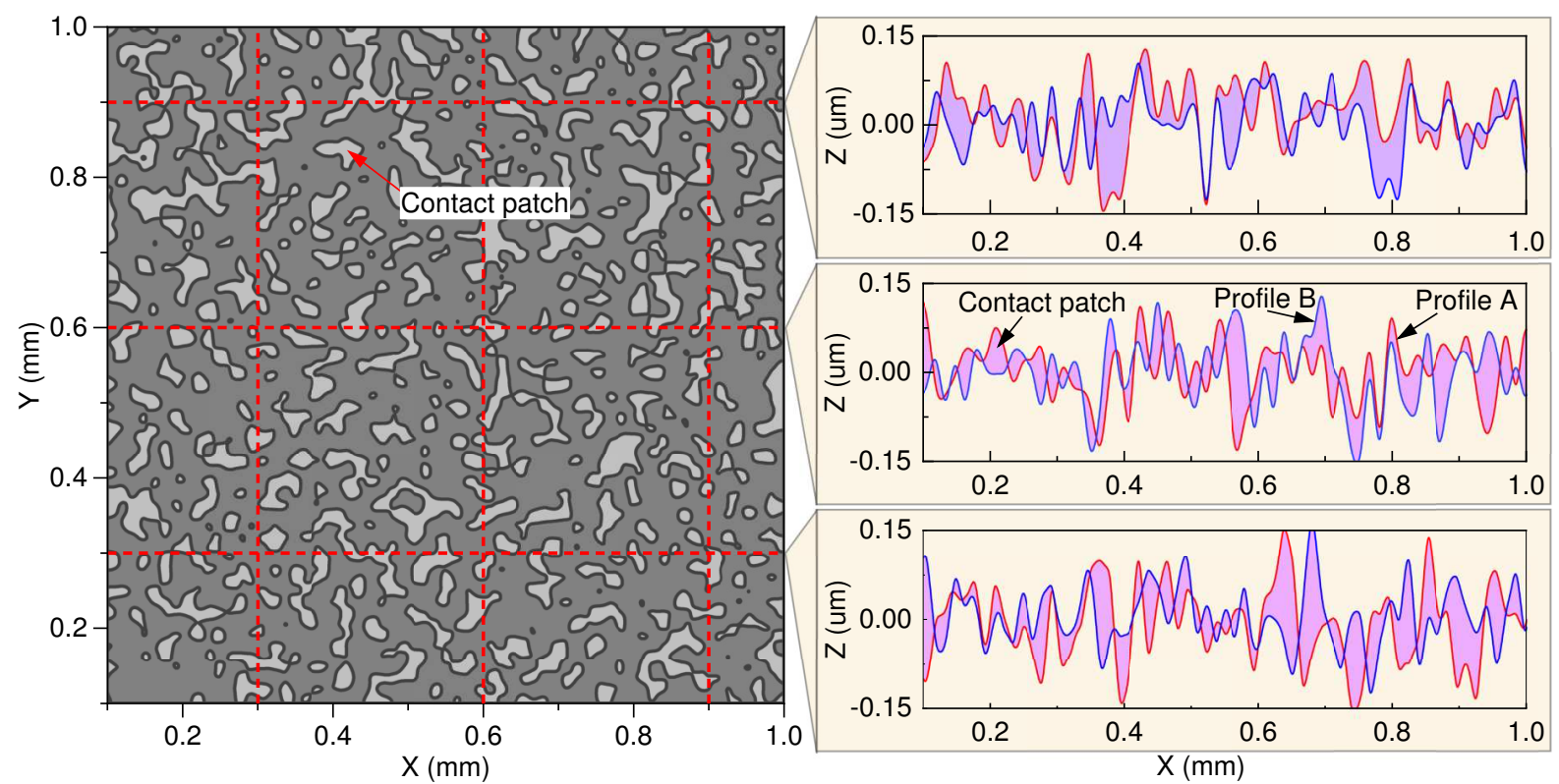

Fig. 15 Schematic diagram of $2 \mathrm{D}$ cross-sectional profile sampling

where $n$ and $m_{j}$ are the number of $2 \mathrm{D}$ sample profiles and asperity in $j$-th profile, respectively. $l_{i j}$ is the length of the $i$-th asperity cut by the $j$-th transversal.

Therefore, the equivalent contact area $S_{e 3 D}$ between the rectangular isotropic rough surface sample can be expressed as:

$$
\begin{aligned}
S_{e 3 D} & =L_{a} N_{u} \times L_{b} N_{u} \times S_{u} \\
& =L_{a} \frac{\sum_{j=1}^{n} N_{j}}{n L_{a}} \times L_{b} \frac{\sum_{j=1}^{n} N_{j}}{n L_{a}} \times\left(\sum_{j=1}^{n} \sum_{i=1}^{m_{j}} \pi\left(l_{i j} / 2\right)^{2}\right) \times \frac{1}{\sum_{j=1}^{n} N_{j}} \\
& =\frac{\pi L_{b}}{4 L_{a}} \sum_{j=1}^{n} N_{j} \sum_{i=1}^{m} l_{i j}^{2}
\end{aligned}
$$

If a certain cross-sectional profile between two rough interfaces can be obtained by image processing methods, and its equivalent contact area between two isotropic 3D rough surfaces can be quickly estimated by Eq.23. Based on this principle, the real contact area between two 3D rough surfaces can be predicted quickly by numerical simulation. Because the computational complexity of this 2D method is much smaller than that of the traditional 3D numerical simulation method, it has a wider range of applications than traditional methods.

\subsection{Effectiveness analysis for the equivalent calculation method}

The equivalent contact area calculated by the proposed method is compared with the existing Equivalent-Plane contact method to illustrate their differences. The calculation principles of the two methods are shown in Fig.16.

The calculation strategy of multiple profiles orthogonal sampling is proposed in the previous section. However, it is not difficult to think that the number of samples may affect the calculation accuracy of the equivalent model. The influence of sampling profiles number on the prediction accuracy of the equivalent calculation method will be specifically studied in this section. Fig.17 is the influence of $2 \mathrm{D}$ profile sampling number on the accuracy of the proposed equivalent calculation method. In the figure, $S_{3 D}$ is the actual contact area between 3D rough surfaces calculated by the image processing method, and $S_{e 3 D}$ is the equivalent 3D contact area obtained by the equivalent calculation method proposed in this paper. "Error" is the error percentage of $S_{e 3 D}$ relative to $S_{3 D}$. It can be seen from Fig. $17 S_{e 3 D} \mathrm{H} 1 \mathrm{~V} 1$ that the equivalent calculation accuracy of an orthogonal sampling line increases rapidly when the real contact area ratio is less than $10 \%$ and greater than $25 \%$. This demonstrates that the proposed equivalent 3D contact area calculated by an orthogonal sampling line may have a large error when the actual contact area is greater than $25 \%$ or less than $10 \%$. Fig. $17 S_{e 3 D} \mathrm{H} 2 \mathrm{~V} 2$ shows that when the real contact area is less than $10 \%$, the error of equivalent 3D contact area calculated by the two sampling lines is still large. However, the error of the equivalent calculation method proposed in this paper can be effectively controlled within $25 \%$ when the real contact area percent is greater than $10 \%$. Fig. $17 S_{e 3 D} \mathrm{H} 3 \mathrm{~V} 3$ show that increasing the number of sampling lines can gradually increase the prediction accuracy of the equivalent calculation method proposed in this paper 


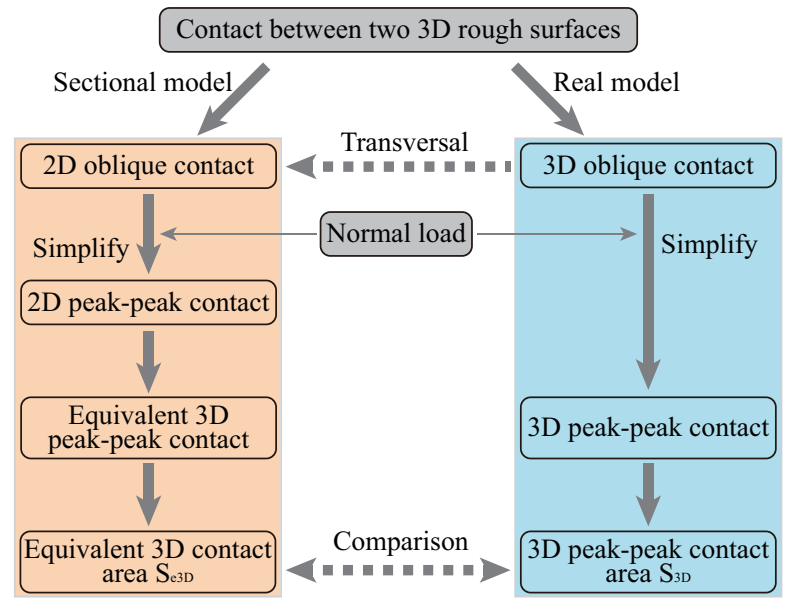

Fig. 16 Comparison between the proposed equivalent method and the existing equivalent method. The yellow shaded area represents the equivalent calculation method proposed in this paper, and the blue shaded area is the existing Equivalent-Plane contact calculation method.

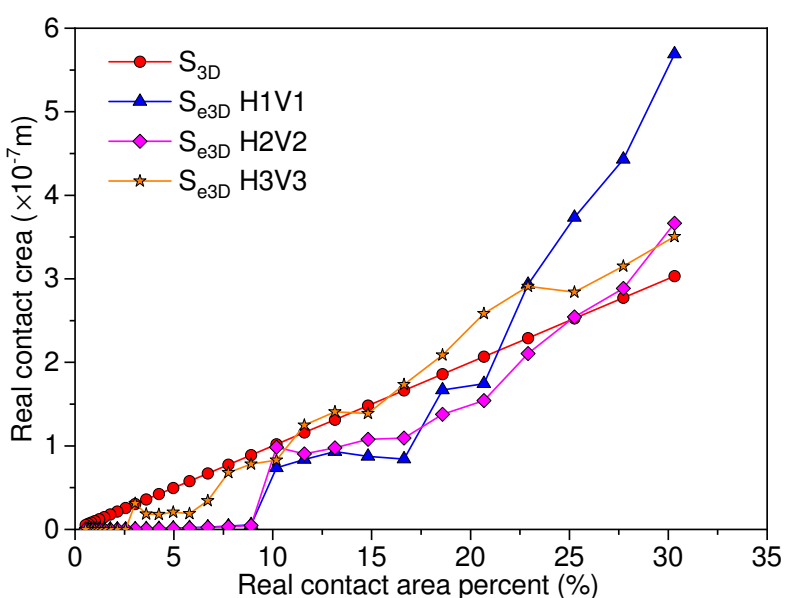

(a)

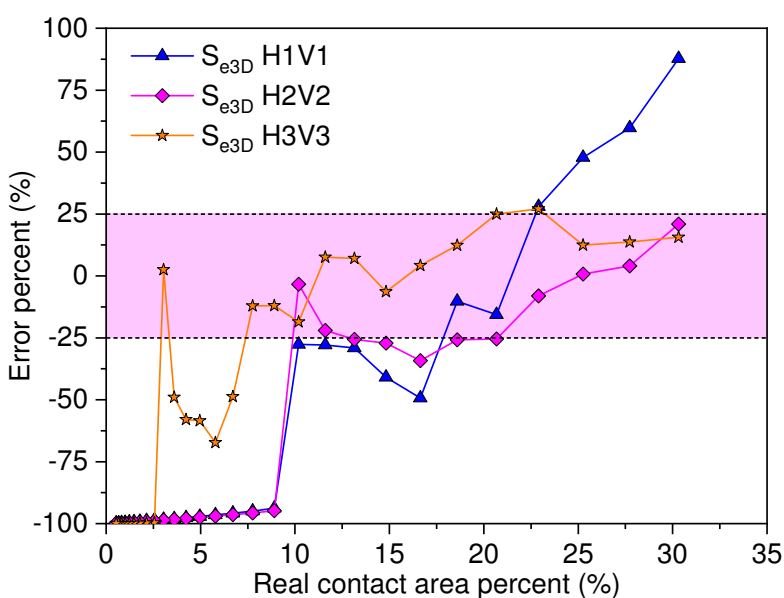

(b)

Fig. 17 Comparison of the calculated equivalent 3D contact area with different numbers of orthogonal sampling lines. $S_{3 D}$ is the real contact area of two 3D rough surfaces obtained by image processing. $S_{e 3 D}$ (H1V1) represents the equivalent 3D contact area calculated by a horizontal and longitudinal sampling line, and $S_{e 3 D}(\mathrm{H} 2 \mathrm{~V} 2)$, and $S_{e 3 D}$ (H3V3) are the equivalent 3D contact areas calculated by two, and three orthogonal sampling lines, respectively.

when the real contact area percent is small, but the prediction accuracy may be reduced when the real contact area percent is larger. This is because the increase in the number of sampling lines makes the sample more uniform and comprehensive when the contact area is small. However, the increase in the number of sampling lines may cause the same asperity is repeatedly sampled by multiple sampling lines when the contact area percent is large, which in turn makes the 3D contact area obtained by the equivalent calculation too large. In addition, it can be seen from Fig.17(b) that the number of sampling lines is not as many as possible. When the contact area is smaller, the more the number of sampling lines, the higher the calculation accuracy. However, when the contact area is larger, the number of sampling lines may decrease the calculation accuracy.

To further verify the equivalent 3D contact area calculation method proposed in this paper, the 300 pairs of rough surfaces are randomly generated using Eq.1, and then the equivalent 3D contact area $S_{e 3 D}$ is calculated using the method based on $2 \mathrm{D}$ cross-sectional profiles proposed in this paper. In addition, the real contact area $S_{3 D}$ of 3D rough surfaces is directly calculated by the method based on 3D image processing proposed in this paper. Since the real contact area between rough surfaces is scale-dependent, the real contact area decreases with the reduction of the resolution scale. To ensure the comparability of the two calculation methods, the resolution must be consistent. The calculation cost of directly calculating the 3D contact area is very high, and the computing power of the computing platform of the research group is limited. Therefore, when the contact area between rough surfaces is calculated, the resolution of the two calculation methods is set to $10^{-6} \mathrm{~m}$. The result is shown in Fig. 18. It can be seen from the figure that the calculation method proposed in this paper increases the error significantly when the 
true contact area percent is less than $5 \%$ or greater than $70 \%$. This is because when the contact area is too small, the two calculation methods with a resolution of $10^{-6} \mathrm{~m}$ have large errors. If it is necessary to improve the calculation accuracy in the equivalent calculation method, the effective measures may include increasing the resolution or increasing the number of $2 \mathrm{D}$ sampling lines in the equivalent calculation method. But if we want to improve the calculation accuracy of the contact area between two 3D rough surfaces by the direct calculation method, there is no other choice but to increase the resolution. This will increase the computational cost exponentially, which is unacceptable in many cases. In addition, the purpose of orthogonal sampling in this paper is to increase the sample length, but the length of the contact surface is mostly larger than the millimeter scale in the real physical world, so the length of the cross-section is theoretically sufficient. In other words, when two rough surfaces are in contact with each other under uniform pressure, Eq.20 can ensure the calculation accuracy of the equivalent 3D contact area.

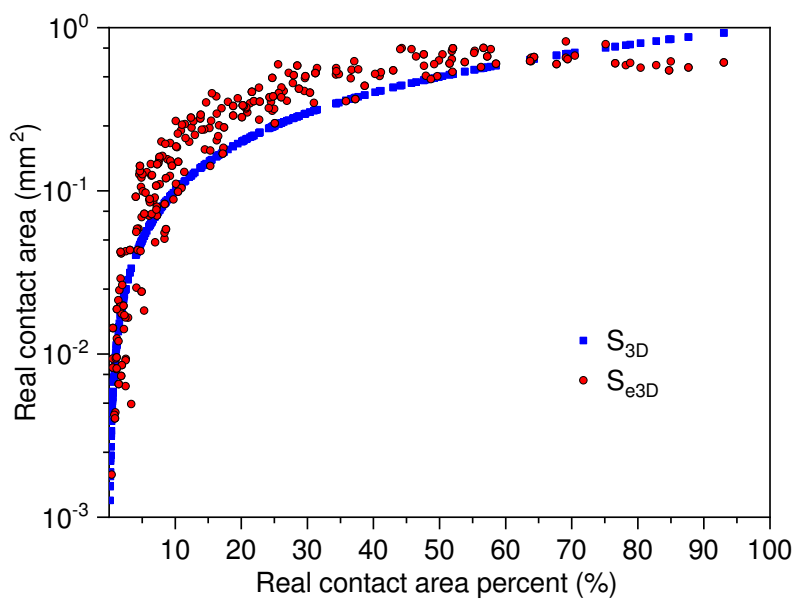

Fig. 18 Comparison of equivalent calculation and direct calculation results for 3D contact area

The advantage of the equivalent 3D contact area calculation method based on the 2D cross-section profile is that no matter whether the resolution is increased or the number of sampling lines is increased, its calculation requirements are linearly increased, so there is no problem of the exponential explosion of calculation cost. This makes it possible for this method to extend the calculation accuracy of the two 3D contact areas to the nanometer level, which is of great significance in dealing with certain microscopic problems. It is obviously difficult to achieve this calculation scale by other numerical methods.

\section{Conclusion}

The real contact area between the mechanical interfaces only occupies a small proportion of the nominal contact area, but it may have a significant impact on the overall stiffness and damping characteristics of the mechanical system. Therefore, it is very important to study the real contact area between the mechanical interfaces.

In this paper, the numerical simulation method is used to calculate the contact area and angle distribution characteristics of the $2 \mathrm{D}$ oblique and peak-peak contact. The research results show that the contact angles between 2D profiles have strong symmetry with respect to zero degrees, then the conclusion is derived that the contact between two $2 \mathrm{D}$ rough surfaces can be simplified to the contact between an equivalent rough surface and a rigid plane when only the normal load is considered. When they have the same normal mechanical properties, the tangential contact forces are canceled each other. In addition, the image processing method is adopted to calculate the contact area between two 3D rough surfaces. Secondly, a calculation method for the oblique contact area and angle between two 3D rough surfaces is proposed based on the projection theorem, and then based on the conclusions drawn from the 2D profile, it is concluded that the contact between 3D rough surfaces can be simplified to contact between an equivalent 3D rough surface and a rigid plane when only normal loads are considered. Thirdly, the relationship between the sampling resolution and the calculation accuracy of the real contact area is analyzed, and the calculation accuracy of the $2 \mathrm{D}$ and $3 \mathrm{D}$ contact area is verified. The analysis results show that when the scale of the asperity to be measured is smaller than the resolution, the actual contact area between asperities measured by numerical simulation methods may be unreliable. The 2D and 3D contact area calculation methods proposed in this article can accurately calculate the true contact area of asperities, whose diameter is larger than the resolution scale. 
Aiming at the problem that it is difficult to accurately calculate the real contact area by the existing simulation methods, and the calculation cost of these methods may sometimes be unacceptable. In this paper, a 3D contact area equivalent calculation method based on $2 \mathrm{D}$ profiles is proposed, and the sampling strategy is designed. Numerical simulation results show that the equivalent calculation method proposed in this paper can effectively calculate the real contact area between two 3D rough surfaces with the contact area percent in the range of 5\%-30\%. When the contact area percent is less than $5 \%$, it can be approximately considered that there is almost no contact between rough surfaces. When the contact area percent is greater than $30 \%$, the contact surface is almost completely yielded, which is rarely present in engineering. Therefore, the equivalent 3D contact area calculation method proposed in this paper is suitable for most engineering situations.

The equivalent 3D contact area calculation method proposed in this paper is low in calculation cost, so the calculation accuracy can be improved through low calculation cost. Therefore, the calculation accuracy of the real contact area between 3D rough surfaces can be extended to nanometers or even smaller scales, which is of great significance for the study of microscopic contact mechanical properties.

\section{References}

[1] Zhang, F., Liu, J., Ding, X., Wang, R.: Experimental and finite element analyses of contact behaviors between non-transparent rough surfaces. Journal of the Mechanics and Physics of Solids 126, 87-100 (2019). https: //doi.org/10.1016/j.jmps.2019.02.004

[2] Mu, X., Sun, Q., Xu, J., Chai, Z., Sun, W., Zhao, B.: Feasibility analysis of the replacement of the actual machining surface by a 3D numerical simulation rough surface. International Journal of Mechanical Sciences 150, 135-144 (2019). https://doi.org/10.1016/j.ijmecsci.2018.10.023

[3] Maleki, I., Wolski, M., Woloszynski, T., Podsiadlo, P., Stachowiak, G.: A comparison of multiscale surface curvature characterization methods for tribological surfaces. Tribology Online 14(1), 8-17 (2019). https://doi. org/10.2474/trol.14.8

[4] Zhang, C., Ren, W.: Modeling of 3d surface morphologies for predicting the mechanical contact behaviors and associated electrical contact resistance. Tribology Letters 69(1), 20 (2021). https://doi.org/10.1007/ s11249-020-01392-9

[5] Lan, G., Sun, W., Zhang, X., Chen, Y., Tan, W., Li, X.: A three-dimensional fractal model of the normal contact characteristics of two contacting rough surfaces. AIP Advances 11(5), 055023 (2021). https://doi.org/ $10.1063 / 5.0045151$

[6] Wang, D., De Boer, G., Neville, A., Ghanbarzadeh, A.: A new numerical model for investigating the effect of surface roughness on the stick and slip of contacting surfaces with identical materials. Tribology International 159, 106947 (2021). https://doi.org/10.1016/j.triboint.2021.106947

[7] Jamshidi, H., Ahmadian, H.: A modified rough interface model considering shear and normal elastic deformation couplings. International Journal of Solids and Structures 203, 57-72 (2020). https://doi.org/10.1016/j. ijsolstr.2020.07.013

[8] Sakakibara, R., Yoshida, I., Nagai, S., Kondo, Y., Yamashita, K.: Surface roughness evaluation method based on roughness parameters in ISO 13565-3 using the least-squares method for running-in wear process analysis of plateau surface. Tribology International 163, 107151 (2021). https://doi.org/10.1016/j.triboint.2021.107151

[9] Mishra, T., de Rooij, M., Schipper, D.J.: The effect of asperity geometry on the wear behaviour in sliding of an elliptical asperity. Wear 470-471, 203615 (2021). https://doi.org/10.1016/j.wear.2021.203615

[10] Ciavarella, M., Joe, J., Papangelo, A., Barber, J.R.: The role of adhesion in contact mechanics. Journal of The Royal Society Interface 16(151), 20180738 (2019). https://doi.org/10.1098/rsif.2018.0738

[11] Xiao, H., Sun, Y., Xu, J.: Investigation into the normal contact stiffness of rough surface in line contact mixed elastohydrodynamic lubrication. Tribology Transactions 61(4), 742-753 (2018). https://doi.org/10. 1080/10402004.2017.1404177 
[12] Pan, W., Li, X., Wang, L., Guo, N., Mu, J.: A normal contact stiffness fractal prediction model of dry-friction rough surface and experimental verification. European Journal of Mechanics - A/Solids 66, 94-102 (2017). https://doi.org/10.1016/j.euromechsol.2017.06.010

[13] Jana, T., Mitra, A., Sahoo, P.: Dynamic contact interactions of fractal surfaces. Applied Surface Science 392, 872-882 (2017). https://doi.org/10.1016/j.apsusc.2016.09.025

[14] Pawlus, P., Reizer, R., Wieczorowski, M.: A review of methods of random surface topography modeling. Tribology International 152, 106530 (2020). https://doi.org/10.1016/j.triboint.2020.106530

[15] Pérez-Ràfols, F., Almqvist, A.: Generating randomly rough surfaces with given height probability distribution and power spectrum. Tribology International 131, 591-604 (2019). https://doi.org/10.1016/j.triboint.2018.11. 020

[16] Yu, X., Sun, Y., Zhao, D., Wu, S.: A revised contact stiffness model of rough curved surfaces based on the length scale. Tribology International 164, 107206 (2021). https://doi.org/10.1016/j.triboint.2021.107206

[17] Li, L., Wang, J., Pei, X., Chu, W., Cai, A.: A modified elastic contact stiffness model considering the deformation of bulk substrate. Journal of Mechanical Science and Technology 34(2), 777-790 (2020). https: //doi.org/10.1007/s12206-020-0126-3

[18] Pan, W., Li, H., Qu, H., Ling, L., Wang, L.: Investigation of tangential contact damping of rough surfaces from the perspective of viscous damping mechanism. Journal of Tribology 143(4), 041501 (2021). https: //doi.org/10.1115/1.4048274

[19] Couto Carneiro, A.M., Pinto Carvalho, R., Andrade Pires, F.M.: Representative contact element size determination for micromechanical contact analysis of self-affine topographies. International Journal of Solids and Structures 206, 262-281 (2020). https://doi.org/10.1016/j.ijsolstr.2020.09.006

[20] Wang, S., Komvopoulos, K.: A fractal theory of the interfacial temperature distribution in the slow sliding regime: Part i - elastic contact and heat transfer analysis. Journal of Tribology 116(4), 812-822 (1994). https: //doi.org/10.1115/1.2927338

[21] Pastewka, L., Robbins, M.O.: Contact area of rough spheres: Large scale simulations and simple scaling laws. Applied Physics Letters 108(22), 221601 (2016). https://doi.org/10.1063/1.4950802

[22] Jacobs, T.D.B., Martini, A.: Measuring and understanding contact area at the nanoscale: A review. Applied Mechanics Reviews 69(6), 060802 (2017). https://doi.org/10.1115/1.4038130

[23] Müser, M., Wang, A.: Contact-patch-size distribution and limits of self-affinity in contacts between randomly rough surfaces. Lubricants $6(4), 85$ (2018). https://doi.org/10.3390/lubricants6040085

[24] Khajeh Salehani, M., van Dokkum, J.S., Irani, N., Nicola, L.: On the load-area relation in rough adhesive contacts. Tribology International 144, 106099 (2020). https://doi.org/10.1016/j.triboint.2019.106099

[25] Zhai, C., Hanaor, D., Gan, Y.: Contact stiffness of multiscale surfaces by truncation analysis. International Journal of Mechanical Sciences 131-132, 305-316 (2017). https://doi.org/10.1016/j.ijmecsci.2017.07.018

[26] Greenwood, J.A., Williamson, J.B.P.: Contact of nominally flat surfaces. Proceedings of the Royal Society of London. Series A. Mathematical and Physical Sciences 295(1442), 300-319 (1966). https://doi.org/10.1098/ rspa.1966.0242

[27] Greenwood, J.A., Tripp, J.H.: The contact of two nominally flat rough surfaces. Proceedings of the Institution of Mechanical Engineers 185(1), 625-633 (1970). https://doi.org/10.1243/PIME_PROC_1970_185_069_02

[28] Sun, Y., Xiao, H., Xu, J.: Contact stiffness ratio of tribological interface using the equivalent thin layer and the micro-slip model. Proceedings of the Institution of Mechanical Engineers, Part C: Journal of Mechanical Engineering Science 234(2), 444-456 (2020). https://doi.org/10.1177/0954406219878758 
[29] Chang, W.R., Etsion, I., Bogy, D.B.: An elastic-plastic model for the contact of rough surfaces. Journal of Tribology 109(2), 257-263 (1987). https://doi.org/10.1115/1.3261348

[30] Brake, M.R.: An analytical elastic-perfectly plastic contact model. International Journal of Solids and Structures 49(22), 3129-3141 (2012). https://doi.org/10.1016/j.ijsolstr.2012.06.013

[31] Whitehouse, D., Archard, J.: The properties of random surfaces of significance in their contact. Proceedings of the Royal Society of London. A. Mathematical and Physical Sciences 316(1524), 97-121 (1970). https: //doi.org/10.1098/rspa.1970.0068

[32] Yuan, Y., Cheng, Y., Liu, K., Gan, L.: A revised majumdar and bushan model of elastoplastic contact between rough surfaces. Applied Surface Science 425, 1138-1157 (2017). https://doi.org/10.1016/j.apsusc.2017.06.294

[33] Mandelbrot, B.B.: Stochastic models for the Earth's relief, the shape and the fractal dimension of the coastlines, and the number-area rule for islands. Proceedings of the National Academy of Sciences 72(10), 3825-3828 (1975). https://doi.org/10.1073/pnas.72.10.3825

[34] M. V., B., Z. V., L.: On the weierstrass-mandelbrot fractal function. Proceedings of the Royal Society of London. A. Mathematical and Physical Sciences 370(1743), 459-484 (1980). https://doi.org/10.1098/rspa. 1980.0044

[35] Mandelbrot, B.B., Wheeler, J.A.: The fractal geometry of nature. American Journal of Physics 51(3), 286-287 (1983). https://doi.org/10.1119/1.13295

[36] Yan, W., Komvopoulos, K.: Contact analysis of elastic-plastic fractal surfaces. Journal of Applied Physics 84(7), 3617-3624 (1998). https://doi.org/10.1063/1.368536

[37] Ausloos, M., Berman, D.H.: A multivariate weierstrass-mandelbrot function. Proceedings of the Royal Society of London. A. Mathematical and Physical Sciences 400(1819), 331-350 (1985). https://doi.org/10.1098/rspa. 1985.0083

[38] Zhang, M., Chen, J., Ma, Y., Shen, L., He, Y., Lin, H.: Fractal reconstruction of rough membrane surface related with membrane fouling in a membrane bioreactor. Bioresource Technology 216, 817-823 (2016). https: //doi.org/10.1016/j.biortech.2016.06.034

[39] Hong, H., Lin, H., Mei, R., Zhou, X., Liao, B.-Q., Zhao, L.: Membrane fouling in a membrane bioreactor: A novel method for membrane surface morphology construction and its application in interaction energy assessment. Journal of Membrane Science 516, 135-143 (2016). https://doi.org/10.1016/j.memsci.2016.06.006

[40] Zhou, H., Long, X., Meng, G., Liu, X.: A stiffness model for bolted joints considering asperity interactions of rough surface contact. Journal of Tribology 144(1), 011501 (2022). https://doi.org/10.1115/1.4050601

[41] Li, X., Li, Z., Jin, S., Zhang, J.: A multi-scale model of real contact area for linear guideway based on the fractal theory. Proceedings of the Institution of Mechanical Engineers, Part C: Journal of Mechanical Engineering Science, 095440622098336 (2021). https://doi.org/10.1177/0954406220983367

[42] Li, L., Tian, H., Yun, Q., Chu, W.: Study on temperature rise distribution of contact surface under cyclic load. Proceedings of the Institution of Mechanical Engineers, Part J: Journal of Engineering Tribology 235(1), 138-148 (2021). https://doi.org/10.1177/1350650120919877

[43] Chen, Q., Xu, F., Liu, P., Fan, H.: Research on fractal model of normal contact stiffness between two spheroidal joint surfaces considering friction factor. Tribology International 97, 253-264 (2016). https://doi.org/10.1016/ j.triboint.2016.01.023

[44] Cheng, R., Jin, Y., Olhofer, M., Sendhoff, B.: A reference vector guided evolutionary algorithm for manyobjective optimization. IEEE Transactions on Evolutionary Computation 20(5), 773-791 (2016). https://doi. org/10.1109/TEVC.2016.2519378

[45] Morag, Y., Etsion, I.: Resolving the contradiction of asperities plastic to elastic mode transition in current 
contact models of fractal rough surfaces. Wear 262(5-6), 624-629 (2007). https://doi.org/10.1016/j.wear.2006. 07.007

[46] Miao, X., Huang, X.: A complete contact model of a fractal rough surface. Wear 309(1-2), 146-151 (2014). https://doi.org/10.1016/j.wear.2013.10.014

[47] Wei, C., Zhu, H., Lang, S.: A modified complete normal contact stiffness model of a fractal surface considering contact friction. Fractals 28(05), 2050081 (2020). https://doi.org/10.1142/S0218348X20500814

[48] Chen, H., Yin, Q., Dong, G., Xie, L., Yin, G.: Stiffness model of fixed joint considering self-affinity and elastoplasticity of asperities. Industrial Lubrication and Tribology 72(1), 128-135 (2019). https://doi.org/10. 1108/ILT-05-2019-0192

[49] Persson, B.N.J.: Contact mechanics for randomly rough surfaces. Surface Science Reports 61(4), 201-227 (2006). https://doi.org/10.1016/j.surfrep.2006.04.001

[50] Afferrante, L., Bottiglione, F., Putignano, C., Persson, B.N.J., Carbone, G.: Elastic contact mechanics of randomly rough surfaces: An assessment of advanced asperity models and persson's theory. Tribology Letters 66(2), 75 (2018). https://doi.org/10.1007/s11249-018-1026-x

[51] Papangelo, A., Hoffmann, N., Ciavarella, M.: Load-separation curves for the contact of self-affine rough surfaces. Scientific Reports 7(1), 6900 (2017). https://doi.org/10.1038/s41598-017-07234-4

[52] Uchidate, M., Yanagi, K., Yoshida, I., Shimizu, T., Iwabuchi, A.: Generation of 3D random topography datasets with periodic boundaries for surface metrology algorithms and measurement standards. Wear 271(3-4), 565570 (2011). https://doi.org/10.1016/j.wear.2010.04.035

[53] Yang, Z., Ding, X., Liu, J., Zhang, F.: Effect of the finite size of generated rough surfaces on the percolation threshold. Proceedings of the Institution of Mechanical Engineers, Part C: Journal of Mechanical Engineering Science 233(16), 5897-5902 (2019). https://doi.org/10.1177/0954406219854470

[54] Yoshida, I., Tsukada, T.: Uncertainty of wavelength limitation due to stylus tip radius for engineering surface texture based on wavelength and amplitude by FFT. Wear 261(11-12), 1225-1231 (2006). https://doi.org/10. $1016 /$ j.wear.2006.03.006

[55] Qu, X., Cai, X., Zhang, M., Lin, H., Leihong, Z., Liao, B.-Q.: A facile method for simulating randomly rough membrane surface associated with interface behaviors. Applied Surface Science 427, 915-921 (2018). https://doi.org/10.1016/j.apsusc.2017.08.013

[56] Noii, N., Aghayan, I.: Characterization of elastic-plastic coated material properties by indentation techniques using optimisation algorithms and finite element analysis. International Journal of Mechanical Sciences 152, 465-480 (2019). https://doi.org/10.1016/j.ijmecsci.2019.01.010

[57] Etsion, I., Kligerman, Y., Kadin, Y.: Unloading of an elastic-plastic loaded spherical contact. International Journal of Solids and Structures 42(13), 3716-3729 (2005). https://doi.org/10.1016/j.ijsolstr.2004.12.006

[58] Megalingam, A., Ramji, K.S.H.: A comparison on deterministic, statistical and statistical with asperity interaction rough surface contact models. Journal of Bio- and Tribo-Corrosion 7(3), 95 (2021). https://doi.org/10. $1007 /$ s40735-021-00526-3

[59] Putignano, C., Afferrante, L., Carbone, G., Demelio, G.: A new efficient numerical method for contact mechanics of rough surfaces. International Journal of Solids and Structures 49(2), 338-343 (2012). https: //doi.org/10.1016/j.ijsolstr.2011.10.009

[60] Campañá, C., Müser, M.H., Robbins, M.O.: Elastic contact between self-affine surfaces: Comparison of numerical stress and contact correlation functions with analytic predictions. Journal of Physics: Condensed Matter 20(35), 354013 (2008). https://doi.org/10.1088/0953-8984/20/35/354013

[61] Prodanov, N., Dapp, W.B., Müser, M.H.: On the contact area and mean gap of rough, elastic contacts: 
Dimensional analysis, numerical corrections, and reference data. Tribology Letters 53(2), 433-448 (2014). https://doi.org/10.1007/s11249-013-0282-z

[62] Pinto Carvalho, R., Couto Carneiro, A.M., Andrade Pires, F.M., Doca, T.: An efficient multiscale strategy to predict the evolution of the real contact area between rough surfaces. Tribology International 165, 107255 (2022). https://doi.org/10.1016/j.triboint.2021.107255

[63] Campañá, C.: Using Green's function molecular dynamics to rationalize the success of asperity models when describing the contact between self-affine surfaces. Physical Review E 78(2), 026110 (2008). https://doi.org/ 10.1103/PhysRevE.78.026110

[64] Wang, G.F., Liang, X.M., Yan, D.: An incremental equivalent circular contact model for rough surfaces. Journal of Tribology 143(8), 081503 (2021). https://doi.org/10.1115/1.4050602

[65] Yastrebov, V.A., Anciaux, G., Molinari, J.-F.: From infinitesimal to full contact between rough surfaces: Evolution of the contact area. International Journal of Solids and Structures 52, 83-102 (2015). https://doi. org/10.1016/j.ijsolstr.2014.09.019

[66] Yastrebov, V.A., Anciaux, G., Molinari, J.-F.: On the accurate computation of the true contact-area in mechanical contact of random rough surfaces. Tribology International 114, 161-171 (2017). https://doi.org/10.1016/ j.triboint.2017.04.023

\section{Statements and Declarations}

Funding. This work was supported by National Natural Science Foundation of China [grant numbers 12072190]; National Major Science and Technology Projects of China [grant numbers 779608000000200007].

Competing Interests. The authors declare that they have no known competing financial interests or personal relationships that could have appeared to influence the work reported in this paper.

Author Contributions. All authors contributed to the study conception and design. Meng Wen: Conceptualization, Formal analysis, Investigation, Methodology, Software, Validation, Writing-original draft, Writing-review \& editing. Zezeng Dai: Investigation, Software, Validation, Visualization, Writing-review \& editing. Jianping Jing: Conceptualization, Methodology, Investigation, Project leader, Writing-review \& editing. Qing Mei: Methodology, Investigation, Writing-review \& editing. Wei Yuan: Methodology, Investigation, Writing-review \& editing. All authors read and approved the final manuscript. 